\title{
PINCEMENT SUR LE SPECTRE ET LE VOLUME EN COURBURE DE RICCI POSITIVE
}

\author{
PAR ERWANN AUBRY ${ }^{1}$
}

\begin{abstract}
RÉSUMÉ. - Nous montrons qu'une variété riemannienne complète de dimension $n$, de courbure Ric $\geqslant$ $n-1$ et dont la $n$-ième valeur propre est proche de $n$ est Gromov-Hausdorff proche de $\left(\mathbb{S}^{n}\right.$, can $)$ et difféomorphe à $\mathbb{S}^{n}$. Ce résultat étend de manière optimale un résultat de P. Petersen [Invent. Math. 138 (1999) 1] (au passage nous comblons le trou annoncé par l'auteur dans l'erratum [Invent. Math. 155 (2004) 223]). Nous montrons également qu'une variété vérifiant l'inégalité Ric $\geqslant n-1$ et de volume proche de $\frac{V_{o l} \mathbb{S}^{n}}{\# \pi_{1}(M)}$ est difféomorphe à un quotient isométrique $\mathbb{S}^{n} / \pi_{1}(M)$ et Gromov-Hausdorff proche de la métrique de courbure 1. Ceci améliore des résultats de T. Colding [Invent. Math. 124 (1996) 175] et T. Yamaguchi [Math. Ann. 284 (1989) 423].
\end{abstract}

(C) 2005 Elsevier SAS

ABSTRACT. - We shall show that a complete Riemannian manifold of dimension $n$ with Ric $\geqslant n-1$ and its $n$-st eigenvalue close to $n$ is both Gromov-Hausdorff close and diffeomorphic to the standard sphere. This extends, in an optimal way, a result of P. Petersen [Invent. Math. 138 (1999) 1] (as a by-product, we fill a gap stated in the erratum [Invent. Math. 155 (2004) 223]). We shall also show that a manifold with Ric $\geqslant n-1$ and volume close to $\frac{\mathrm{Vol}^{n}}{\# \pi_{1}(M)}$ is both Gromov-Hausdorff close and diffeomorphic to a space form $\mathbb{S}^{n} / \pi_{1}(M)$. This extends results of T. Colding [Invent. Math. 124 (1996) 175] and T. Yamaguchi [Math. Ann. 284 (1989) 423].

(C) 2005 Elsevier SAS

\section{Introduction}

Dans cet article, $M$ désigne par défaut une variété riemannienne complète de dimension $n$ et de courbure de Ricci Ric $\geqslant(n-1)$. Dans l'ensemble de ces variétés, la sphère $\mathbb{S}^{n}$ (munie de sa métrique canonique) est un point extrémal pour de nombreux invariants riemanniens. Des théorèmes de comparaison classiques dus à S. Myers, R. Bishop, A. Lichnerowicz, S. Cheng et M. Obata (voir par exemple [20]) astreignent les variétés de cet ensemble à vérifier Diam $M \leqslant$ Diam $\mathbb{S}^{n}=\pi$ (et donc $M$ est compacte), $\pi_{1}(M)$ est fini, $\operatorname{Vol} M \leqslant \operatorname{Vol} \mathbb{S}^{n}, \operatorname{Rad} M \leqslant \operatorname{Rad} \mathbb{S}^{n}=\pi$ (où Rad désigne le rayon couvrant, i.e. le rayon de la plus petite boule géodésique qui recouvre toute la variété), $\lambda_{1}(M) \geqslant \lambda_{1}\left(\mathbb{S}^{n}\right)=n$ (où $0=\lambda_{0}(M)<\lambda_{1}(M) \leqslant \lambda_{2}(M) \leqslant \cdots$ désigne le spectre du laplacien sur la variété, compté avec multiplicité). De plus, si l'égalité est réalisée dans une de ces inégalités, alors la variété $M$ est isométrique à la sphère canonique $\mathbb{S}^{n}$ (auquel cas, $n$ est une valeur propre de $M$ de multiplicité $n+1$ ).

$\mathrm{Au}$ vu de ces faits, il est naturel de rechercher les propriétés (topologiques, différentiables ou métriques) de $\mathbb{S}^{n}$ qui sont conservées par les variétés riemanniennes de courbure

\footnotetext{
${ }^{1}$ Travaux en partie financés par la bourse FNRS Suisse nº 20-101469. 
Ric $\geqslant n-1$ pour lesquelles l'un des invariants riemanniens considérés plus haut prend une valeur suffisamment proche de sa valeur extrémale. Ce genre de problèmes de stabilité est dorénavant classique lorsqu'on suppose en plus que la courbure sectionnelle des variétés considérées reste minorée par une constante fixée. La recherche d'analogues à ces résultats lorsque seule la courbure de Ricci est supposée minorée est encouragée par le célèbre résultat de Gromov qui implique que l'ensemble des variétés riemanniennes complètes de courbure de Ricci minorée par $(n-1)$ est précompact pour la distance de Gromov-Hausdorff (nous renvoyons le lecteur à $[12,20]$ pour la définition et les propriétés usuelles de la distance de Gromov-Hausdorff sur l'ensemble des variétés riemanniennes complètes), et connaît un essort récent grâce aux travaux pionniers de G. Perelman [18], T. Colding et J. Cheeger [8,9,6,7].

Dans [8,9], Colding montre que les trois conditions suivantes sont équivalentes (toujours sous l'hypothèse Ric $\geqslant n-1)$ :

(1) Vol $M$ est proche de $\mathrm{Vol} \mathbb{S}^{n}$,

(2) $\operatorname{Rad} M$ est proche de $\pi$,

(3) $M$ est Gromov-Hausdorff proche de $\left(\mathbb{S}^{n}\right.$, can $)$.

P. Petersen a par la suite prouvé que la condition (2) est équivalente à ce que les $n+1$ premières valeurs propres (non nulles) du laplacien de $M$ soient proches de $n$ (voir [16]). De plus, ces quatre conditions impliquent que $M$ est difféomorphe à $\mathbb{S}^{n}$, d'après le résultat suivant.

ThÉORÈme (J. Cheeger et T. Colding [6]). - Soient $K$ un réel fixé et $\left(M_{p}^{n}, g_{p}\right)_{p \in \mathbb{N}}$ une suite de variétés riemanniennes complètes vérifiant $\operatorname{Ric}_{g_{p}} \geqslant K$ et qui converge au sens de GromovHausdorff vers une variété riemannienne $\left(M_{\infty}^{n}, g\right)$ compacte (de même dimension). Alors $M_{p}$ est difféomorphe à $M_{\infty}$ pour p assez grand.

Le but de cet article est de démontrer trois résultats de stabilité nouveaux. Le premier est une version optimisée (et quantitative) du résultat énoncé dans [16].

THÉORÈME 1. - Soit $\left(M^{n}, g\right)$ une variété riemannienne complète de courbure de Ricci minorée par $n-1$. Il existe des constantes $\epsilon(n)>0, \beta(n)>0$ et $C(n)>0$ telles que si les $n$ premières valeurs propres non nulles du laplacien de $M$ sont inférieures à $n+\epsilon(n)$ alors la distance de Gromov-Hausdorff entre $\mathbb{S}^{n}$ et $M$ est majorée par $C(n)\left(\lambda_{n}-n\right)^{\beta(n)}$ et $M$ est donc difféomorphe à $\mathbb{S}^{n}$.

Remarquons que nous ne demandons que le contrôle des $n$ premières valeurs propres non nulles dans cet énoncé. De plus, dans notre schéma de preuve, une approximation de Hausdorff à valeur dans $\mathbb{S}^{n}$ est explicitement construite, ce qui rend calculable la valeur $\epsilon(n)$ à partir de laquelle elle existe.

Remarque. - Le théorème 1 est optimal en ce qui concerne la première conclusion, puisqu'on construit dans cet article une suite de métriques $g_{k}$ sur $\mathbb{S}^{n}$ telle que $\operatorname{Ric}\left(g_{k}\right) \geqslant n-1, \lambda_{i}\left(g_{k}\right) \rightarrow n$ pour $1 \leqslant i \leqslant n-1$ et telle que la suite $\left(\mathbb{S}^{n}, g_{k}\right)$ tende (en distance de Gromov-Hausdorff) vers la demi-sphère de dimension $n-1$ munie de sa métrique canonique. En revanche, le problème de savoir à partir de quelle valeur $k$, l'hypothèse $\lambda_{k}(M) \leqslant n(1+\epsilon(n))$ implique que la variété $M^{n}$ est difféomorphe à $\mathbb{S}^{n}$ est encore un problème ouvert (il résulte du théorème 1 et de contreexemples dus à M. Anderson [1] et Y. Otsu [15] que cette valeur est comprise entre 2 et $n$ ).

Notons que J. Bertrand démontre dans [4] que les $k$ premières valeurs propres non nulles de $M$ sont proches de $n$ si et seulement s'il existe une partie de $M$ Hausdorff proche de $\mathbb{S}^{k-1}$. De ceci et du théorème 1 découle le corollaire suivant.

Corollaire 2. - Il existe des constantes $\epsilon(n)>0, \beta(n)>0$ et $C(n)>0$ telles que, si $M$ contient une partie A qui, munie de la distance induite, est à distance de Gromov-Hausdorff

$4^{\text {e }}$ SÉRIE - TOME $38-2005-\mathrm{N}^{\circ} 3$ 
de $\left(\mathbb{S}^{n-1}\right.$, can) inférieure à $\epsilon \leqslant \epsilon(n)$, alors $M$ est difféomorphe à $\mathbb{S}^{n}$ et $M$ est à distance de Gromov-Hausdorff de $\mathbb{S}^{n}$ inférieure à $C(n) \epsilon^{\beta(n)}$.

Remarque. - Ce corollaire est encore valable s'il existe une partie $A$ de $M$ qui est GromovHausdorff proche du sous-ensemble $\left\{ \pm e_{1}, \ldots, \pm e_{n}\right\}$ de $\mathbb{S}^{n}$ (où $\left(e_{1}, \ldots, e_{n+1}\right)$ est une base orthonormée de $\mathbb{R}^{n+1}$ ).

Avant d'énoncer les autres résultats principaux de cet article, rappelons que le volume d'une variété $M$ de courbure Ric $\geqslant n-1$ est majoré par $\operatorname{Vol} \mathbb{S}^{n} / \# \pi_{1}(M)$, l'égalité étant atteinte si et seulement si $\pi_{1}(M)$ est un sous-groupe (fini) de $\mathrm{O}(n+1)$ agissant librement sur $\mathbb{S}^{n}$ et si la variété riemannienne $M$ est l'espace quotient $\left(\mathbb{S}^{n}, \mathrm{can}\right) / \pi_{1}(M)$ (résultat obtenu en appliquant au revêtement universel riemannien de $\left(M^{n}, g\right)$ les théorèmes de R. Bishop et de $\mathrm{S}$. Cheng cités plus haut; voir [20]). De même, si $M$ est non orientable, alors son volume est majoré par $\operatorname{Vol}\left(\mathbb{S}^{n}\right.$, can $) / 2$, l'égalité étant atteinte si et seulement si la dimension $n$ est paire et $M=\mathbb{P}^{n} \mathbb{R}$.

Dans le cas où $M$ est de volume presque maximal, nous prouvons les deux résultats suivants (notons que le second améliore à la fois un résultat de T. Colding [8], qui ne traite que le cas où le volume de $M$ est proche de celui de $\mathbb{S}^{n}$, et un résultat de T. Yamaguchi [22] qui suppose de plus que la courbure sectionnelle de $M$ est minorée par une constante $-K^{2}$ ).

THÉORÈME 3. - Il existe des constantes $\epsilon(n)>0, \beta(n)>0$ et $C(n)>0$ telles que, si $M$ est non simplement-connexe et vérifie $\mathrm{Vol} M \geqslant \frac{\mathrm{Vol}^{n}}{2}(1-\epsilon(n))$, alors la distance de GromovHausdorff entre $M$ et $\mathbb{P}^{n}(\mathbb{R})$ est majorée par

$$
C(n)\left(\frac{\operatorname{Vol} \mathbb{S}^{n}}{2}-\operatorname{Vol} M\right)^{\beta(n)} \quad \text { et } \quad \text { M est difféomorphe à } \mathbb{P}^{n}(\mathbb{R}) \text {. }
$$

On remarquera que ce résultat s'applique au cas non-orientable et que, dans ce cas, la conclusion est que $n$ est pair, $M$ est difféomorphe à $\mathbb{P}^{n}(\mathbb{R})$ et Gromov-Hausdorff proche de $\mathbb{P}^{n}(\mathbb{R})$. Dans le cas où le groupe fondamental est de cardinal quelconque, on a le résultat suivant.

ThÉORÈme 4. - Soit $k \in \mathbb{N}^{*}$. Il existe des constantes $\epsilon(n, k)>0, \beta(n)>0$ et $C(n)>0$ telles que si $M$ vérifie:

$$
\operatorname{Vol} M \geqslant \frac{\operatorname{Vol} \mathbb{S}^{n}}{k}(1-\epsilon(n, k)) \quad \text { et } \quad \# \pi_{1}(M) \geqslant k,
$$

alors $\pi_{1}(M)$ est un sous-groupe (de cardinal $k$ ) de $\mathrm{O}(n+1)$ agissant librement sur $\mathbb{S}^{n}$, la distance de Gromov-Hausdorff entre $M$ et $\mathbb{S}^{n} / \pi_{1}(M)$ est majorée par $C(n)\left[\frac{\mathrm{Vol}^{n}}{k}-\operatorname{Vol} M\right]^{\beta(n)}$ et $M$ est difféomorphe à l'espace quotient $\mathbb{S}^{n} / \pi_{1}(M)$.

En remarquant que tout quotient de la sphère canonique par un groupe d'isométries non trivial (agissant librement sur $\mathbb{S}^{n}$ ) est de diamètre majoré par $\frac{\pi}{2}$, on obtient, en courbure de Ricci minorée par $n-1$, le corollaire suivant.

COROllaire 5. - Il existe des constantes $\epsilon(n)>0$ et $\delta(n)>0$ telles que si $M$ vérifie les deux inégalités :

$$
\operatorname{Vol} M \geqslant \frac{\operatorname{Vol} \mathbb{S}^{n}}{2}(1-\delta(n)) \quad \text { et } \quad \operatorname{Diam} M \geqslant \frac{\pi}{2}+\epsilon(n),
$$

alors $M$ est simplement connexe.

Dans [7], les auteurs démontrent que la fonctionnelle $i$ ème valeur propre est continue pour la distance de Gromov-Hausdorff sur l'ensemble des variétés riemanniennes complètes de 
dimension $n$, de diamètre majoré par $D$ et de courbure de Ricci minorée par $-(n-1)$. Comme les espaces modèles $\left(\mathbb{S}^{n}, \mathrm{can}\right) / \pi_{1}(M)$ obtenus sous les hypothèses du théorème 4 sont en nombre fini, on en déduit l'amélioration suivante du théorème de Lichnerowicz.

COROllaire 6. - Soient $k \geqslant 2$ et $p$ des entiers et $\epsilon>0$ un réel. Il existe une constante $\eta(\epsilon, n, k, p)>0$ telle que si $M$ vérifie :

$$
\operatorname{Vol} M \geqslant \frac{\operatorname{Vol} \mathbb{S}^{n}}{k}(1-\eta(\epsilon, n, k, p)) \quad \text { et } \quad \# \pi_{1}(M) \geqslant k,
$$

alors $\lambda_{i}\left(M^{n}, g\right) \geqslant(1-\epsilon) \lambda_{i}\left(\left(\mathbb{S}^{n}\right.\right.$, can $\left.) / \pi_{1}(M)\right) \geqslant 2 n(1-\epsilon)$ pour tout $i \leqslant p$ (voir [13] pour un calcul du spectre des variétés de courbure constante égale à 1$)$.

En particulier, on en déduit un énoncé équivalent au corollaire 5 où l'hypothèse sur le diamètre est remplacée par l'hypothèse $\lambda_{1} \leqslant 2 n(1-\epsilon(n))$.

Il découle des arguments utilisés dans la démonstration de notre théorème 4 une autre amélioration des théorèmes de Lichnerowicz et de Myers où le pincement requis sur le volume ne dépend pas de $\# \pi_{1}(M)$.

COROLlAIRE 7. - Il existe une constante $\delta(n)>0$ telle que si $M$ est non simplement connexe alors :

$$
\begin{gathered}
\lambda_{1}(M) \geqslant n\left(\frac{\# \pi_{1}(M) \operatorname{Vol} M}{\operatorname{Vol} \mathbb{S}^{n}}+\delta(n)\right), \\
\operatorname{Diam} M \leqslant(\pi-\delta(n))\left[2-\frac{\# \pi_{1}(M) \operatorname{Vol} M}{\operatorname{Vol} \mathbb{S}^{n}}\right] .
\end{gathered}
$$

Remarque. - La démonstration de ce dernier corollaire est plus simple que celle du résultat de [7] utilisé pour le corollaire 6. Toutefois la conclusion est plus décevante car les bornes obtenues sont a priori très éloignées des valeurs réalisées par les espaces modèles du théorème 4 . Les corollaires 6 et 7 améliorent l'inégalité de Lichnerowicz dans le cas de variétés de courbure de Ricci minorée par $n-1$, non simplement connexes et de volume presque maximal. On peut se demander si le pincement sur le volume est vraiment nécessaire pour qu'une telle amélioration de l'inégalité de Lichnerowicz ait lieu (il est troublant de constater que les seuls exemples connus de variétés de courbure de Ricci minorée par $n-1$ et de $\lambda_{1}$ proche de $n$ sont simplement connexes ; voir $[1,15])$.

PLAN DE L'ARTICLE : Dans la section 2 nous établissons les estimées analytiques sur les fonctions propres de $M$ nécessaires à nos démonstrations, dans la section 3 nous nous plaçons dans le cas où $M$ admet $n+1$ valeurs propres proches de $n$ et, en suivant $[11,16]$, nous construisons à l'aide de ces fonctions propres une approximation de Hausdorff $\Phi$ de $M$ dans $\mathbb{S}^{n}$ de degré \pm 1 (nous prouvons ce dernier fait, qui manquait dans [16]; voir l'erratum [17]). Cependant, notre méthode de preuve s'appuiera sur des arguments différents de ceux proposés dans $[11,16]$. Dans la section 4 , nous montrons qu' un sous-groupe fini d'isométries de $M$ admet une action isométrique sur $\mathbb{S}^{n}$ qui rend $\Phi$ équivariante et que, si la première action est libre, alors la seconde l'est aussi. Dans la section 5, nous donnons la preuve des théorèmes 3 et 4 et du corollaire 7. Pour cela, nous montrons que nos variétés sont Hausdorff proches d'un quotient de la sphère par un groupe fini d'isométries, mais pour pouvoir conclure, il faut toutefois montrer que ce quotient est une variété non singulière. Pour ce point délicat, T. Yamaguchi utilise dans [22] une hypothèse supplémentaire de minoration de la courbure sectionnelle, qui implique une minoration de la systole de $M$. Nous nous passons de cette hypothèse grâce au résultat de la section 4 (quant à la technique de T. Colding pour traiter le cas $k=0$ du théorème 4 , elle

$4^{\mathrm{e}}$ SÉRIE - TOME $38-2005-\mathrm{N}^{\circ} 3$ 
utilise de manière essentielle le fait que l'espace modèle est dans ce cas de rayon couvrant $\pi$; cette technique ne peut pas s'adapter trivialement pour démontrer le théorème 4). En section 6 nous prouvons le théorème 1 et décrivons en section 7 la famille de contre-exemples qui prouve l'optimalité du théorème 1 .

\section{Valeurs propres proches de $n$ et fonctions propres associées}

\section{Fibré augmenté de E. Ruh [19]}

Notons $E \rightarrow M$ le fibré vectoriel obtenu comme somme de Withney de $T M$ et d'un fibré trivial en droite (on notera $E=T M \oplus \mathbb{R} e$ ). Le produit scalaire et la connexion linéaire suivants munissent $E$ d'une structure de fibré riemannien :

$$
\begin{aligned}
& \langle X+f e, Y+h e\rangle_{E}=g(X, Y)+f h \\
& D_{Z}^{E}(X+f e)=D_{Z}^{M} X+f Z+(d f(Z)-g(Z, X)) \cdot e
\end{aligned}
$$

où on a noté $g$ la métrique de $M$ et $D^{M}$ sa connexion de Levi-Civita.

Remarque. - Le fibré normal de $\mathbb{S}^{n}$ dans $\mathbb{R}^{n+1}$ est un fibré trivial en droites dont la somme de Withney avec $T \mathbb{S}^{n}$, munie de la métrique décrite plus haut n'est autre que le fibré trivial $E=\mathbb{S}^{n} \times\left(\mathbb{R}^{n+1}\right.$, can $)$. Rappelons que les fonctions propres de $\mathbb{S}^{n}$ associées à la valeur propre $n$ sont de la forme $f(x)=\langle u, x\rangle_{\mathbb{R}^{n+1}}$, où $u$ est un vecteur quelconque de $\mathbb{R}^{n+1}$. Notons $e(x)=x$; alors la correspondance $f \mapsto \nabla f+f . e=u$ donne une identification naturelle entre l'espace des fonctions propres associées à la valeur propre $n$ de $\mathbb{S}^{n}$ et les sections parallèles du fibré $E$. On va montrer dans la suite que cette correspondance se généralise à toutes les variétés de courbure de Ricci presque minorée par $n-1$ : le laplacien de $M$ sur les fonctions aura autant de valeurs propres proches de $n$ que le laplacien brut $\bar{\triangle}^{E}$ sur $E$ aura de valeurs propres proches de 0 (proposition 10) et les sections de $E$ de la forme $\nabla f+f e$, où $f$ est une fonction propre associée à une valeur propre proche de $n$, seront presque «parallèles» (lemme 11). Inversement les fonctions de la forme $\langle S, e\rangle_{E}$, où les $S$ sont des sections propres associées à de petites valeurs propres seront presque des fonctions propres associées à des valeurs propres proches de $n$.

\section{Opérateur $\triangle_{\mathrm{sph}}$}

Dans la suite, on note $\pi$ la projection orthogonale de E sur son sous-fibré TM et A la symétrie orthogonale d'hyperplan TM. On définit un champ d'endomorphismes symétriques sur $E$ en posant $\operatorname{Ric}^{\prime}(S)=\operatorname{Ric}_{M}(\pi(S))-(n-1) \pi(S)$. On notera par la suite $\triangle_{\text {sph }}$ l'opérateur $\bar{\triangle}^{E}+\mathrm{Ric}^{\prime}$

Sections $S_{f}=\nabla f+f . e$

Le lemme suivant sera fondamental pour relier les fonctions propres de $M$ aux sections propres de $\bar{\triangle}^{E}$.

Lemme 8. - Soit $f: M \rightarrow \mathbb{R}$ telle que $\triangle f=\lambda f$. On pose $S_{f}=\nabla f+f$.e, on a alors la relation $\triangle_{\mathrm{sph}}\left(S_{f}\right)=(\lambda-n) A\left(S_{f}\right)$.

Preuve. - Un calcul direct donne :

$$
\bar{\triangle}^{E}\left(S_{f}\right)(x)=\bar{\triangle}_{M} \nabla f-\nabla f+n f . e-\triangle f . e,
$$


où $\bar{\triangle}_{M}$ est le laplacien brut de $T M$. Enfin, l'opérateur $\bar{\triangle}_{M}+\operatorname{Ric}_{M}$ n'est autre que l'opérateur de Hodge $\triangle_{H}$ sur les champs de vecteurs, qui commute avec $\nabla$, d'où la relation annoncée.

\section{Correspondance fonctions/sections propres}

Nous aurons besoin d'une minoration de la première valeur propre $\lambda_{1}^{1}$ du laplacien de Hodge sur les 1-formes de $M$. L'inégalité suivante découle d'une variante de la méthode de Lichnerowicz. Nous en donnons une preuve succincte.

LEMME 9. - Soit $M$ vérifiant $\operatorname{Ric} \geqslant n-1$; alors $\lambda_{1}^{1}(M) \geqslant n$ (respectivement la première valeur propre du laplacien de Hodge, restreint aux 1-formes co-fermées est minorée par $2(n-1))$.

Preuve. - Soit $\alpha \in \Lambda^{1}(M)$. En décomposant orthogonalement $D \alpha$ en partie antisymétrique $\frac{d \alpha}{2}$, partie symétrique sans trace et partie scalaire $-\frac{\delta \alpha}{n} g$, on obtient $|D \alpha|^{2} \geqslant \frac{|d \alpha|^{2}}{2}+\frac{(\delta \alpha)^{2}}{n}$. En intégrant la formule de Bochner on obtient :

$$
(\triangle \alpha, \alpha)=\|D \alpha\|_{2}^{2}+\int_{M} \operatorname{Ric}(\alpha, \alpha) \geqslant \frac{\|d \alpha\|_{2}^{2}}{2}+\frac{\|\delta \alpha\|_{2}^{2}}{n}+(n-1)\|\alpha\|_{2}^{2} .
$$

Si $d \alpha=0$, alors $\|\delta \alpha\|_{2}^{2}=(\triangle \alpha, \alpha)$ et donc $(\triangle \alpha, \alpha) \geqslant n\|\alpha\|^{2}$. Si $\delta \alpha=0$, alors $\|d \alpha\|_{2}^{2}=(\triangle \alpha, \alpha)$ et donc $(\triangle \alpha, \alpha) \geqslant 2(n-1)\|\alpha\|^{2}$.

Nous en déduisons la proposition suivante, qui affirme que $\triangle, \triangle^{E}$ et $\triangle_{\mathrm{sph}}$ ont le même nombre de petites valeurs propres.

Proposition 10. - Il existe des constantes $\alpha^{\prime}(n)>\alpha(n)>0$ (explicitement calculables) telles que, si $M$ est une variété complète vérifiant $\mathrm{Ric} \geqslant n-1$, alors :

(i) $\lambda_{n+2}(M) \geqslant n+\alpha^{\prime}(n)$ et $\lambda_{n+2}\left(\triangle_{\mathrm{sph}}\right) \geqslant \lambda_{n+2}\left(\bar{\triangle}^{E}\right) \geqslant \alpha^{\prime}(n)$.

(ii) Pour tout entier $k, 0 \leqslant \lambda_{k}\left(\triangle^{E}\right) \leqslant \lambda_{k}\left(\triangle_{\mathrm{sph}}\right) \leqslant\left(\lambda_{k}(M)-n\right)$.

Réciproquement, si $\lambda_{k}\left(\bar{\triangle}^{E}\right) \leqslant \alpha(n)$ alors

$$
\lambda_{k}(M) \leqslant n+200 n^{2} \sqrt{\lambda_{k}\left(\bar{\triangle}^{E}\right)} .
$$

Preuve. - La première série d'inégalités annoncée en (ii) découle directement de la positivité du potentiel Ric' ${ }^{\prime}$, du lemme 8 et du principe du min-max.

Pour finir de démontrer (ii), notons $\left(S_{i}\right)_{1 \leqslant i \leqslant k}$ une famille $L^{2}$-orthonormée de sections propres (associées aux $k$ premières valeurs propres) de $\bar{\triangle}^{E}$. Notons $\mathcal{E}$ l'espace vectoriel engendré par les sections $\left(S_{i}\right)_{1 \leqslant i \leqslant k}$, muni du produit scalaire $L^{2}$ de $E$, et $\mathcal{F}=\Psi(\mathcal{E})$, où $\Psi(S)=\langle S, e\rangle_{E}$ (notons que $\mathcal{F}$ est engendré par les fonctions $f_{i}=\left\langle S_{i}, e\right\rangle_{E}$ ). Nous allons conclure en appliquant le principe du min-max à $\mathcal{F}$ :

- Les fonctions de $\mathcal{F}$ sont d'intégrale nulle car $\bar{\triangle}^{E}$ est auto-adjoint, $\bar{\triangle}^{E} e=$ n.e (lemme 8, avec $f=1), \bar{\triangle}^{E}\left(S_{i}\right)=\lambda_{i} S_{i}$ et $\lambda_{i} \neq n$, d'où $\frac{1}{\operatorname{Vol} M} \int_{M} f_{i}=\frac{1}{\operatorname{Vol} M} \int_{M}\left\langle S_{i}, e\right\rangle_{E}=0$.

- $\Psi$ est une application injective, et donc $\mathcal{F}$ est un espace de fonctions de dimension $k$ : si $S \in \operatorname{Ker} \Psi \cap \mathcal{E}$, alors $S=X \in \Gamma(M)$ et $\left\|D^{E} S\right\|_{2}^{2} \leqslant \alpha(n)\|S\|_{2}^{2}<\|S\|_{2}^{2}$. Or $D_{Y}^{E} X=$ $D_{Y}^{M} X-g(X, Y) e$, et donc $\left\|D^{E} S\right\|_{2}^{2}=\left\|D^{M} X\right\|_{2}^{2}+\|X\|_{2}^{2} \geqslant\|X\|_{2}^{2}=\|S\|_{2}^{2}$. On en déduit que $S=0$.

- Il ne reste plus qu'à montrer que le quotient de Rayleigh des fonctions de $\mathcal{F}$ est presque plus petit que $n$. Soit donc $f \in \mathcal{F} \backslash\{0\}$. Il existe donc $\alpha \in \Lambda^{1}(M)$ tel que $S=\alpha^{\#}+f$.e $\in \mathcal{E}$, ce qui donne :

$4^{\mathrm{e}}$ SÉRIE - TOME $38-2005-\mathrm{N}^{\circ} 3$ 


$$
\begin{aligned}
& \left\|D^{M} \alpha^{\#}+f \operatorname{Id}_{T M}-(d f-\alpha) \cdot e\right\|_{2}^{2} \\
& \quad=\left\|D^{E} S\right\|_{2}^{2} \leqslant \lambda_{k}\left(\bar{\triangle}^{E}\right)\|S\|_{2}^{2}=\lambda_{k}\left(\bar{\triangle}^{E}\right)\left(\|\alpha\|_{2}^{2}+\|f\|_{2}^{2}\right) .
\end{aligned}
$$

Dont on déduit les deux inégalités suivantes :

$$
\begin{array}{r}
\left\|D^{M} \alpha+f g\right\|_{2}^{2} \leqslant \lambda_{k}\left(\bar{\triangle}^{E}\right)\left(\|\alpha\|_{2}^{2}+\|f\|_{2}^{2}\right), \\
\|d f-\alpha\|_{2}^{2} \leqslant \lambda_{k}\left(\bar{\triangle}^{E}\right)\left(\|\alpha\|_{2}^{2}+\|f\|_{2}^{2}\right) .
\end{array}
$$

La deuxième de ces inégalités donne l'inégalité :

$$
\left|\frac{\|d f\|_{2}}{\|f\|_{2}}-\frac{\|\alpha\|_{2}}{\|f\|_{2}}\right| \leqslant \sqrt{\lambda_{k}\left(\bar{\triangle}^{E}\right)}\left(1+\frac{\|\alpha\|_{2}}{\|f\|_{2}}\right) .
$$

On est donc ramené à montrer que le rapport $\|\alpha\|_{2}^{2} /\|f\|_{2}^{2}$ est presque majoré par $n$. Or, en procédant comme dans la démonstration du lemme 9 :

$$
\frac{\|d \alpha\|_{2}^{2}}{2}+\left\|f-\frac{\delta \alpha}{n}\right\|_{2}^{2} \leqslant\left\|D^{M} \alpha+f g\right\|_{2}^{2} \leqslant \lambda_{k}\left(\bar{\triangle}^{E}\right)\left(\|\alpha\|_{2}^{2}+\|f\|_{2}^{2}\right) .
$$

On en déduit :

$$
\begin{aligned}
(\triangle \alpha, \alpha) & =\|d \alpha\|_{2}^{2}+\|\delta \alpha\|_{2}^{2} \\
& \leqslant 5 n^{2} \sqrt{\lambda_{k}\left(\bar{\triangle}^{E}\right)}\left(\|\alpha\|_{2}^{2}+\|f\|_{2}^{2}\right)+\left(1+\sqrt{\lambda_{k}\left(\bar{\triangle}^{E}\right)}\right) n^{2}\|f\|_{2}^{2} .
\end{aligned}
$$

Enfin, d'après le lemme 9 , on a $(\triangle \alpha, \alpha) \geqslant n\|\alpha\|_{2}^{2}$. En combinant les deux dernières inégalités, on obtient $\|\alpha\|_{2}^{2} \leqslant\left(1+12 n \sqrt{\lambda_{k}\left(\bar{\triangle}^{E}\right)}\right) n\|f\|_{2}^{2}$, dès que $\alpha(n) \leqslant \frac{1}{36 n^{4}}$, ce qui permet de conclure :

(i) sera démontré pour l'opérateur $\bar{\triangle}^{E}$ dans la section suivante. Pour les autres opérateurs, cela découle alors de (ii).

Remarque. - On pourrait renforcer l'analogie avec le cas de $\mathbb{S}^{n}$ décrit précédemment en montrant que, si $f$ est une combinaison linéaire des fonctions propres de $M$ associées à des valeurs propres proches de $n$, alors $S_{f}$ est $L^{2}$-proche d'une combinaison linéaire des sections propres de $\triangle^{E}$ associées à de petites valeurs propres (la réciproque est aussi vraie en remplaçant $f \mapsto S_{f}$ par $\left.S \mapsto\langle S, e\rangle_{E}\right)$.

\section{Estimées sur les fonctions propres}

Soit $\left(\sqrt{n+1} \cdot f_{i}\right)_{1 \leqslant i \leqslant k}$ une famille $L^{2}$-orthonormée de fonctions propres de $M$ associées à des valeurs propres $0<\lambda_{1} \leqslant \cdots \leqslant \lambda_{k} \leqslant n+\epsilon$. On lui associe la famille $L^{2}$-orthogonale $\left(S_{i}\right)_{1 \leqslant i \leqslant k}$ de sections du fibré $E$ définies par $S_{i}=\nabla f_{i}+f_{i} e$. On obtient alors les estimées analytiques et géométriques suivantes.

LEMME 11. - Il existe des constantes $\alpha(n)>0$ et $C(n)>0$ (explicitement calculables) telles que si $M$ vérifie $\lambda_{k} \leqslant n+\epsilon($ avec $\epsilon \leqslant \alpha(n))$, alors :

(i) $\left\|\sum_{i=1}^{k} \alpha_{i} S_{i}\right\|_{\infty} \leqslant(1+C(n) \sqrt{\epsilon})\left\|\sum_{i=1}^{k} \alpha_{i} S_{i}\right\|_{2}$, pour tout $\left(\alpha_{i}\right) \in \mathbb{R}^{k}$.

(ii) Il existe un sous-ensemble $M_{\epsilon}$ de $M$ tel que : $\operatorname{Vol} M_{\epsilon} \geqslant\left(1-C(n) \epsilon^{1 / 4}\right) \operatorname{Vol} M$ $\left|\left\langle S_{i}(x), S_{j}(x)\right\rangle_{E}-\delta_{i j}\right| \leqslant C(n) \epsilon^{1 / 4}$ pour tout $x \in M_{\epsilon}$ et tout couple $(i, j)$.

Remarque. - Si la famille $\left(S_{i}\right)_{1 \leqslant i \leqslant k}$ est une famille $L^{2}$-orthonormée de sections propres d'un opérateur $\bar{\triangle}^{E}+V$ à potentiel $V$ positif associées à des valeurs propres 
$\lambda_{1}\left(\bar{\triangle}^{E}+V\right) \leqslant \cdots \leqslant \lambda_{k}\left(\bar{\triangle}^{E}+V\right) \leqslant \epsilon($ avec $\epsilon \leqslant \alpha(n))$, alors les propriétés (i) et (ii) sont encore valables (la preuve qui suit s'adapte facilement).

Preuve. - D'après le lemme 8 , la famille de sections $\left(\sqrt{\frac{n+1}{\lambda_{i}+1}} S_{i}\right)$ est $L^{2}$-orthonormée et vérifie $\triangle_{\mathrm{sph}} S_{i}=\left(\lambda_{i}-n\right) A\left(S_{i}\right)$ pour tout indice $i \leqslant k$. Soient $E_{k}$ l'espace vectoriel engendré par $\left(S_{i}\right)_{1 \leqslant i \leqslant k}$ et $A_{p}=\operatorname{supp}_{S \in E_{k} \backslash\{0\}}\|S\|_{p} /\|S\|_{2}$, où $\left.\left.p \in\right] 1,+\infty\right]$.

Soit $S \in E_{k} \backslash\{0\}$; posons $u=\sqrt{|S|^{2}+\epsilon^{2}}$ pour $\epsilon>0$. Une technique à la Kato nous donne l'inégalité :

$$
\begin{aligned}
u \triangle u & =\frac{1}{2} \triangle u^{2}+|d u|^{2}=\frac{1}{2} \triangle|S|^{2}+\frac{\left|\langle D S, S\rangle_{E}\right|^{2}}{\sqrt{|S|^{2}+\epsilon^{2}}} \\
& \leqslant \frac{1}{2} \triangle|S|^{2}+|D S|^{2}=\left\langle\bar{\triangle}^{E} S, S\right\rangle_{E} \leqslant\left\langle\triangle_{\mathrm{sph}} S, S\right\rangle_{E} \leqslant\left|\triangle_{\mathrm{sph}} S\right| u .
\end{aligned}
$$

On en déduit que, pour tout réel $p>1 / 2$ :

$$
\left\|d\left(u^{p}\right)\right\|_{2}^{2}=\frac{p^{2}}{2 p-1} \int_{M}(u \triangle u) u^{2 p-2} \leqslant \frac{p^{2}}{2 p-1}\left[\left\|\triangle_{\mathrm{sph}} S\right\|_{2 p}\|u\|_{2 p}^{2 p-1}\right] .
$$

En appliquant l'inégalité de Sobolev $\|f\|_{\frac{2 n}{n-2}}^{2} \leqslant C(n)\|d f\|_{2}^{2}+\|f\|_{2}^{2}$ donnée par [14] à la fonction $u^{p}$ et en faisant tendre $\epsilon$ vers 0 , on obtient :

$$
\|S\|_{\frac{2 p n}{n-2}}^{p} \leqslant \frac{C(n) p}{\sqrt{2 p-1}} \sqrt{\left\|\triangle_{\mathrm{sph}} S\right\|_{2 p}\|S\|_{2 p}^{2 p-1}}+\|S\|_{2 p}^{p}
$$

(cette inégalité reste valable en dimension 2 en remplaçant $n$ par 4). Or $E_{k}$ est un espace stable par $A \triangle_{\mathrm{sph}}$ et $A$ est une isométrie, on a donc :

$$
\left\|\triangle_{\mathrm{sph}} S\right\|_{2 p}=\left\|A \triangle_{\mathrm{sph}} S\right\|_{2 p} \leqslant A_{2 p}\left\|A \triangle_{\mathrm{sph}} S\right\|_{2} \leqslant A_{2 p}\left(\lambda_{k}-n\right) .\|S\|_{2} .
$$

On en déduit l'inégalité $A_{\frac{2 p n}{n-2}} \leqslant\left(1+\frac{C(n) p}{\sqrt{2 p-1}}\left(\lambda_{k}-n\right)^{1 / 2}\right)^{1 / p} A_{2 p}$, et donc $A_{\infty} \leqslant \prod_{j=0}^{\infty}\left(1+\frac{C(n) \nu^{j}}{\sqrt{2 \nu^{j}-1}}\left(\lambda_{k}-n\right)^{1 / 2}\right)^{1 / \nu^{j}}$ en posant $\nu=\frac{n}{(n-2)}$. Enfin, en utilisant la concavité de la fonction log, on obtient $A_{\infty} \leqslant(1+C(n) \sqrt{\epsilon})$.

Nous allons maintenant prouver que l'inégalité (i) implique la propriété (ii). Posons $M_{\epsilon}$ l'ensemble des points $x \in M$ où :

$$
\begin{gathered}
\left|S_{i}(x)+S_{j}(x)\right|_{E}^{2} \geqslant 2\left(1-\epsilon^{1 / 4}\right), \quad\left|S_{i}(x)-S_{j}(x)\right|_{E}^{2} \geqslant 2\left(1-\epsilon^{1 / 4}\right), \\
\left|S_{i}(x)\right|_{E}^{2} \geqslant 1-\epsilon^{1 / 4} \quad \forall i<j .
\end{gathered}
$$

D'après (i) (et en posant $h=\sum_{i<j}\left|S_{i}+S_{j}\right|_{E}^{2}+\left|S_{i}-S_{j}\right|_{E}^{2}+\sum_{i} 2\left|S_{i}\right|_{E}^{2}$ ) :

$$
\begin{aligned}
2 k^{2} & =\frac{1}{\operatorname{Vol} M} \int_{M} h=\frac{1}{\operatorname{Vol} M} \int_{M_{\epsilon}} h+\frac{1}{\operatorname{Vol} M} \int_{M \backslash M_{\epsilon}} h \\
& \leqslant \frac{\operatorname{Vol} M_{\epsilon}}{\operatorname{Vol} M}\left(\sum_{i<j}\left\|S_{i}+S_{j}\right\|_{\infty}^{2}+\left\|S_{i}-S_{j}\right\|_{\infty}^{2}+\sum_{i} 2\left\|S_{i}\right\|_{\infty}^{2}\right)
\end{aligned}
$$

$4^{\mathrm{e}}$ SÉRIE - TOME $38-2005-\mathrm{N}^{\circ} 3$ 


$$
\begin{aligned}
& +\left(k^{2}-1\right)\left(1-\frac{\operatorname{Vol} M_{\epsilon}}{\operatorname{Vol} M}\right) \max _{1 \leqslant i<j \leqslant k}\left[\left\|S_{i}+S_{j}\right\|_{\infty}^{2},\left\|S_{i}-S_{j}\right\|_{\infty}^{2}, 2\left\|S_{i}\right\|_{\infty}^{2}\right] \\
& +2\left(1-\frac{\operatorname{Vol} M_{\epsilon}}{\operatorname{Vol} M}\right)\left(1-\epsilon^{1 / 4}\right) \\
& \leqslant 2 k^{2}(1+C(n) \sqrt{\epsilon}) \frac{\operatorname{Vol} M_{\epsilon}}{\operatorname{Vol} M} \\
& +\left[2\left(k^{2}-1\right)(1+C(n) \sqrt{\epsilon})+2\left(1-\epsilon^{1 / 4}\right)\right]\left(1-\frac{\operatorname{Vol} M_{\epsilon}}{\operatorname{Vol} M}\right) .
\end{aligned}
$$

On en déduit $\frac{\operatorname{Vol} M_{\epsilon}}{\operatorname{Vol} M} \geqslant 1-\frac{k^{2} C(n) \epsilon^{1 / 4}}{1+C(n) \epsilon^{1 / 4}} \geqslant 1-k^{2} C(n) \epsilon^{1 / 4}$, pour $\epsilon \leqslant \alpha(k, n)$ assez petit. D'après (i), on a pour tout $x \in M_{\epsilon}$ et tout $i \neq j$ :

$$
\begin{aligned}
\left|\left\langle S_{i}(x), S_{j}(x)\right\rangle_{E}\right| & =\left|\frac{\left|S_{i}(x)+S_{j}(x)\right|^{2}-\left|S_{i}(x)-S_{j}(x)\right|^{2}}{4}\right| \\
& \leqslant \frac{2(1+C(n) \sqrt{\epsilon})-2\left(1-\epsilon^{1 / 4}\right)}{4} \leqslant C^{\prime}(n) \epsilon^{1 / 4} .
\end{aligned}
$$

De même, $\left|\left\|S_{i}(x)\right\|_{E}^{2}-1\right| \leqslant C^{\prime}(n) \epsilon^{1 / 4}$. Supposons $k>n+1$ : en appliquant ce qui précède à la famille $\left(S_{i}\right)_{1 \leqslant i \leqslant n+2}$, on obtient que $\left|\left\langle S_{i}, S_{j}\right\rangle-\delta_{i j}\right| \leqslant C(n) \epsilon^{1 / 4}$ sur $M_{\epsilon}$. Mais alors, il existe au moins un point $x$ de $M$ où la famille $\left(S_{i}(x)\right)_{1 \leqslant i \leqslant n+2}$ est de rang $n+2$, ce qui est impossible car $E$ est de rang $n+1$. On en déduit d'abord que $k \leqslant n+1$ (et donc le (i) de la proposition 10), puis la propriété (ii) annoncée.

\section{Variétés admettant $n+1$ petites valeurs propres}

Dans cette section nous étudions les variétés complètes vérifiant Ric $\geqslant(n-1)$ et $\lambda_{n+1} \leqslant n+$ $\alpha(n)$. Fixons une famille $\left(\sqrt{n+1} \cdot f_{i}\right)_{1 \leqslant i \leqslant n+1} L^{2}$-orthonormée de fonctions propres associées aux valeurs propres $n \leqslant \lambda_{1} \leqslant \cdots \leqslant \lambda_{n+1} \leqslant n+\epsilon$ et considérons l'application :

$$
\begin{aligned}
\Phi: M & \rightarrow \mathbb{S}^{n} \hookrightarrow \mathbb{R}^{n+1} \\
x & \mapsto \frac{1}{\left(\sum_{j} f_{j}(x)^{2}\right)^{1 / 2}} \cdot\left(f_{1}(x), \ldots, f_{n+1}(x)\right) .
\end{aligned}
$$

Nous démontrons dans cette section la proposition suivante.

Proposition 12. - Il existe des constantes $\alpha(n)>0$ et $C(n)>0$ (explicitement calculables) telles que si $M$ est une variété complète qui vérifie $\mathrm{Ric} \geqslant(n-1)$ et $\lambda_{n+1} \leqslant n+\epsilon$ (pour $\epsilon \leqslant \alpha(n))$; alors :

$$
\operatorname{Vol} M \geqslant\left(1-C(n) \epsilon^{\frac{1}{2(n+1)}}\right) \operatorname{Vol} \mathbb{S}^{n}
$$

et l'application $\Phi$ est une approximation de Hausdorff à valeur dans $\mathbb{S}^{n}$ de degré \pm 1 . Plus précisément, pour tout couple de points $(x, y)$ de $M$, on a :

$$
\left|d_{\mathbb{S}^{n}}(\Phi(x), \Phi(y))-d_{M}(x, y)\right| \leqslant C(n) \epsilon^{\frac{1}{384(n+1)^{3}}} .
$$

Remarque. - Notre schéma de preuve est une adaptation de celui de P. Petersen dans [16] (voir aussi [2]). Toutefois, nous corrigeons l'erreur faite par P. Petersen sur le calcul du degré de $\Phi$ (voir l'erratum [17]) ce qui est indispensable pour notre démonstration des résultats annoncés en introduction. Pour la démonstration du fait que $\Phi$ est une approximation de Hausdorff, nous 
renvoyons à l'article [16] de P. Petersen (noter que d'après ce qui suit, $\Phi$ est surjective). On pourra regarder aussi [3], où la même propriété est démontrée sous des hypothèses de pincement intégral de la courbure de Ricci passant sous $n-1$ et la forme du majorant de la distance de Gromov-Hausdorff est explicitement calculée.

\section{$\Phi$ est bien définie sous nos hypothèses}

Cela découle du lemme suivant.

LEMME 13. - Il existe des constantes $\alpha(n)>0$ et $C(n)>0$ (explicitement calculables) telles que si $\lambda_{n+1} \leqslant n+\epsilon($ avec $\epsilon \leqslant \alpha(n))$, on $a:\left\|\sum_{i=1}^{n+1} f_{i}^{2}-1\right\|_{\infty} \leqslant C(n) \epsilon^{\frac{1}{2(n+1)}}$.

Preuve. - Soit $x_{0}$ un point quelconque de $M$. En appliquant le lemme 11 avec $\alpha_{i}=f_{i}\left(x_{0}\right)$, on obtient :

$$
\begin{aligned}
& \left(\sum_{i=1}^{n+1} f_{i}\left(x_{0}\right) f_{i}\right)^{2}+\left(\sum_{i=1}^{n+1} f_{i}\left(x_{0}\right) \nabla f_{i}\right)^{2} \\
& \quad \leqslant(1+C(n) \sqrt{\epsilon})^{2}\left\|\sum_{i=1}^{n+1} f_{i}\left(x_{0}\right) S_{i}\right\|_{2}^{2} \leqslant(1+C(n) \sqrt{\epsilon}) \sum_{i=1}^{n+1} f_{i}\left(x_{0}\right)^{2} .
\end{aligned}
$$

On en déduit que la fonction $h=\sum_{i=1}^{n+1} f_{i}^{2}$ vérifie les inégalités $\|h\|_{1}=1$, $\|h\|_{\infty} \leqslant\left(1+C^{\prime}(n) \sqrt{\epsilon}\right)$ et $\|d h\|_{\infty} \leqslant C^{\prime}(n)$. Le résultat annoncé découle alors d'un second lemme.

LEMME 14. - Soient $\left(M^{n}, g\right)$ compacte et vérifiant $\mathrm{Ric} \geqslant 0$ et $h: M \rightarrow \mathbb{R}$ une fonction lipschitzienne; alors, pour tout point $x$ de $M$, on a :

$$
|h|(x) \geqslant\|h\|_{\infty}-2\left(\operatorname{Diam} M\|d h\|_{\infty}\right)^{n /(n+1)}\left(\|h\|_{\infty}-\|h\|_{1}\right)^{1 /(n+1)} .
$$

Preuve. - On peut supposer $\|h\|_{1}>0$. Soit $x \in M$ :

$$
\begin{aligned}
\|h\|_{1} & =\frac{1}{\operatorname{Vol} M} \int_{B(x, \eta)}|h|+\frac{1}{\operatorname{Vol} M} \int_{M \backslash B(x, \eta)}|h| \\
& \leqslant \frac{\operatorname{Vol} B(x, \eta)}{\operatorname{Vol} M}\left(|h(x)|+\|d h\|_{\infty} \eta\right)+\left(1-\frac{\operatorname{Vol} B(x, \eta)}{\operatorname{Vol} M}\right)\|h\|_{\infty} .
\end{aligned}
$$

On en déduit que $\|h\|_{\infty}-\|h\|_{1} \geqslant \frac{\operatorname{Vol} B(x, \eta)}{\operatorname{Vol} M}\left(\|h\|_{\infty}-|h(x)|-\|d h\|_{\infty} \eta\right)$. Posons $\eta=$ $\frac{\|h\|_{\infty}-|h(x)|}{2\|d h\|_{\infty}}>0$. Le théorème de Bishop-Gromov donne alors :

$$
\|h\|_{\infty}-\|h\|_{1} \geqslant \frac{\left(\|h\|_{\infty}-|h(x)|\right)^{n+1}}{2^{n+1}\|d h\|_{\infty}^{n}(\operatorname{Diam} M)^{n}} .
$$

\section{Calcul de $d \Phi$}

Soit $x$ un point de $M$; alors $d_{x} \Phi$ est une application de $T_{x} M$ dans $T_{\Phi(x)} \mathbb{S}^{n} \subset \mathbb{R}^{n+1}$. On note ${ }^{t} d_{x} \Phi$ l'application transposée de $d_{x} \Phi$ vu comme une application de $\left(T_{x} M, g_{x}\right)$ dans $\mathbb{R}^{n+1}$ muni de son produit scalaire canonique, qu'on notera $\langle\cdot, \cdot\rangle_{\mathbb{R}^{n+1}}$. Si $\left(\varepsilon_{i}\right)_{1 \leqslant i \leqslant n+1}$ est la base canonique 
de $\mathbb{R}^{n+1}$ alors

$$
{ }^{t} d_{x} \Phi\left(\varepsilon_{i}\right)=\frac{\nabla f_{i}-\Phi_{i} \sum_{k=1}^{n+1} \Phi_{k} \cdot \nabla f_{k}}{\left(\sum_{j} f_{j}^{2}\right)^{1 / 2}}=\frac{S_{i}-\Phi_{i} \sum_{j=1}^{n+1} \Phi_{j} S_{j}}{\left(\sum_{j} f_{j}^{2}\right)^{1 / 2}},
$$

où on a posé

$$
\Phi_{i}=\frac{f_{i}}{\left(\sum_{j} f_{j}^{2}\right)^{1 / 2}}
$$

( $T M$ est vu comme un sous-fibré de $E$ ). En particulier, pour $v$ dans $T_{\Phi(x)} \mathbb{S}^{n}\left(=\Phi(x)^{\perp}\right)$, on a

$$
{ }^{t} d_{x} \Phi(v)=\frac{\sum_{i=1}^{n+1} v_{i} S_{i}}{\left(\sum f_{j}^{2}\right)^{1 / 2}}
$$

\section{$\Phi$ est presque contractante}

Les lemmes 11 et 13 nous donnent alors le résultat suivant.

LEMME 15. - Si $M$ vérifie $\mathrm{Ric} \geqslant n-1$ et si $\lambda_{n+1} \leqslant n+\epsilon($ avec $\epsilon \leqslant \alpha(n))$, alors $\|d \Phi\|_{\infty} \leqslant$ $1+C(n) \epsilon^{\frac{1}{2(n+1)}}$.

Si $M$ est orientable, alors $\operatorname{deg} \Phi= \pm 1$

Soient $x$ un point de $M$ et $\left(X_{i}\right)$ une base orthonormée directe de $T_{x} M$. Pour tout $x \in M$, on munit $E_{x}$ de l'orientation induite par $T M$ (i.e. telle que $\left(\widetilde{X}_{i}\right)_{1 \leqslant i \leqslant n+1}=\left(X_{1}, \ldots, X_{n}, e\right)$ soit une base directe de $\left.E_{x}\right)$. On note $L_{x}: \mathbb{R}^{n+1} \rightarrow E_{x}$ l'application définie par $L_{x}(v)=\sum_{i=1}^{n+1} v_{i} S_{i}(x)$ et $h(x)=\operatorname{det} L_{x}$ (le déterminant étant calculé relativement à des bases orthonormées directes des espaces de départ et d'arrivée). En calculant $h$ dans une base orthonormée directe de $\mathbb{R}^{n+1}$ de la forme $\left(e_{1}, \ldots, e_{n}, \Phi(x)\right)$, on obtient :

$$
h(x)=\left(\sum_{j} f_{j}^{2}(x)\right)^{(n+1) / 2} \operatorname{det} d_{x} \Phi .
$$

Commençons par estimer $\|h\|_{2}$. On a $\|h\|_{\infty}^{2} \leqslant(1+C(n) \sqrt{\epsilon})$ car $h^{2}(x) \leqslant\left|L_{x}\right|^{2 n}$ et d'après le lemme 11(i), on a :

$$
\left|L_{x}(v)\right|_{E}^{2} \leqslant(1+C(n) \sqrt{\epsilon})\left\|\sum_{i=1}^{n+1} v_{i} S_{i}\right\|_{2}^{2} \leqslant(1+C(n) \sqrt{\epsilon})\|v\|_{\mathbb{R}^{n+1}}^{2},
$$

pour tout $v \in \mathbb{R}^{n+1}$. Par ailleurs, pour tout couple $(u, v)$ de vecteurs de $\mathbb{R}^{n+1}$, on a :

$$
\begin{aligned}
& \left|\left\langle{ }^{t} L_{x} \circ L_{x}(u), v\right\rangle_{\mathbb{R}^{n+1}}-\langle u, v\rangle_{\mathbb{R}^{n+1}}\right| \\
& \quad=\left|\sum_{i j} u_{i} v_{j}\left(\left\langle S_{i}, S_{j}\right\rangle_{E}-\delta_{i j}\right)\right| \\
& \quad \leqslant \max _{i j}\left|\left\langle S_{i}, S_{j}\right\rangle_{E}-\delta_{i j}\right|\|u\|_{\mathbb{R}^{n+1}}\|v\|_{\mathbb{R}^{n+1}}
\end{aligned}
$$

et donc, d'après le lemme 11(ii), $\left\|^{t} L_{x} \circ L_{x}-\operatorname{Id}_{T_{\Phi(x)} \mathbb{S}^{n}}\right\| \leqslant C(n) \epsilon^{1 / 4}$, pour tout point $x$ du sousensemble $M_{\epsilon}$, i.e. $\left|h^{2}(x)-1\right| \leqslant C(p, n) \epsilon^{1 / 4}$ sur $M_{\epsilon}$. On obtient alors l'estimée : 


$$
\begin{aligned}
& \left|\frac{1}{\operatorname{Vol} M} \int_{M} h^{2}-1\right| \\
& \quad \leqslant\left|\frac{1}{\operatorname{Vol} M} \int_{M_{\epsilon}}\left(h^{2}-1\right)\right|+\left(1-\frac{\operatorname{Vol} M_{\epsilon}}{\operatorname{Vol} M}\right) \max \left(1,\left\|h^{2}\right\|_{\infty}-1\right) \\
& \leqslant C(n) \epsilon^{1 / 4} .
\end{aligned}
$$

Pour obtenir le degré de $\Phi$, c'est l'intégrale $\frac{1}{\operatorname{Vol} M} \int_{M} h$ que l'on doit estimer. Nous allons utiliser l'inégalité de Poincaré pour montrer que la moyenne de $h$ est proche de sa norme $L^{2}$. Pour cela, il faut montrer que la norme $L^{2}$ de $d h$ est petite :

En prenant la base canonique de $\mathbb{R}^{n+1}$ comme base orthonormée au départ, et une base orthonormée directe quelconque $\left(\widetilde{X}_{i}(x)\right)$ de $E_{x}$ à l'arrivée, on obtient $h(x)=\operatorname{det}\left(\left\langle\widetilde{X}_{i}, S_{j}\right\rangle_{E}\right)$. Soient $x$ un point donné de $M, X \in T_{x} M$ et $\gamma_{X}$ la géodésique passant par $x$ avec le vecteur vitesse $X$. On note $\left(\widetilde{X}_{i}\right)$ le repère transporté parallèlement le long de $\gamma_{X}$ de $\left(\widetilde{X}_{i}(x)\right)$. Alors $d_{x} h(X)=X \cdot\left(\operatorname{det}\left(\left\langle\widetilde{X}_{i}, S_{j}\right\rangle_{E}\right)_{i j}\right)$ est égal à :

$$
\sum_{j=1}^{n+1} \operatorname{det}\left(\begin{array}{ccccc}
\left\langle\widetilde{X}_{1}, S_{1}\right\rangle & \ldots & \left\langle\widetilde{X}_{1}, D_{X}^{E} S_{j}\right\rangle & \ldots & \left\langle\widetilde{X}_{1}, S_{n+1}\right\rangle \\
\left|\tilde{X}_{n+1}, S_{1}\right\rangle & \ldots & \left\langle\widetilde{X}_{n+1}, D_{X}^{E} S_{j}\right\rangle & \ldots & \left\langle\widetilde{X}_{n+1}, S_{n+1}\right\rangle
\end{array}\right) .
$$

D'où $\left|d_{x} h(X)\right| \leqslant C(n) \max _{i}\left\|S_{i}\right\|_{\infty}^{n} \max _{i}\left|D_{X}^{E} S_{i}(x)\right|_{E}$. Le lemme 11(i), donne donc :

$$
\|d h\|_{2}^{2}=\frac{1}{\operatorname{Vol} M} \int_{M}|d h|^{2} \leqslant C(n) \max _{i} \frac{1}{\operatorname{Vol} M} \int\left|D^{E} S_{i}\right|^{2}(x) \leqslant C(n) \epsilon,
$$

la dernière inégalité découle de la preuve du lemme 10(ii).

Or $\lambda_{1} \geqslant n$ et donc $0 \leqslant\|h\|_{2}^{2}-\left(\frac{1}{\operatorname{Vol} M} \int_{M} h\right)^{2} \leqslant C(n) \epsilon$. On déduit donc de l'estimée précédente sur $\|h\|_{2}^{2}$ que :

$$
1-C(n) \epsilon^{1 / 4} \leqslant\left(\frac{1}{\operatorname{Vol} M} \int_{M} h\right)^{2} \leqslant 1+C(n) \epsilon^{1 / 4}
$$

Pour conclure, on a $\operatorname{deg} \Phi \operatorname{Vol} \mathbb{S}^{n}=\int_{M} \operatorname{det} d \Phi=\int_{M}\left(\sum_{k} f_{k}^{2}\right)^{-(n+1) / 2} h$. Le lemme 13 et la majoration de $\|h\|_{\infty}$ donnent alors $\| \operatorname{deg} \Phi\left|\frac{\operatorname{Vol}_{\mathbb{S}^{n}}}{\operatorname{Vol} M}-1\right| \leqslant C(n) \epsilon^{\frac{1}{2(n+1)}}$. Comme $\operatorname{deg} \Phi$ est un entier et que $\operatorname{Vol} M \leqslant \operatorname{Vol} \mathbb{S}^{n}$, on obtient $\operatorname{deg} \Phi= \pm 1$ et $\operatorname{Vol} M \geqslant \operatorname{Vol} \mathbb{S}^{n}\left(1-C(n) \epsilon^{\frac{1}{2(n+1)}}\right)$.

\section{Si $\lambda_{n+1}(M) \leqslant n+\epsilon(n)$ alors $M$ est orientable}

Si $M^{n}$ est non-orientable, on construit l'application $\Phi$ à partir des fonctions propres $\left(f_{i}\right)_{1 \leqslant i \leqslant n+1}$ et on note $\pi: \widetilde{M} \rightarrow M$ le revêtement riemannien orientable de $M$. Soit $\widetilde{\Phi}=$ $\Phi \circ \pi: \widetilde{M} \rightarrow \mathbb{S}^{n}$. On a $\operatorname{deg}_{2} \widetilde{\Phi}=\operatorname{deg}_{2} \Phi \operatorname{deg}_{2} \pi=0$ (où $\operatorname{deg}_{2}$ désigne le degré modulo 2 ), et donc $\widetilde{\Phi}$ est de degré orientable pair. Cependant, $\left(\widetilde{M}^{n}, \tilde{g}\right)$ vérifie aussi Ric $\geqslant(n-1)$ et les fonctions $\tilde{f}_{i}=f_{i} \circ \pi$ sont des fonctions propres de $\left(\widetilde{M}^{n}, \tilde{g}\right)$ associées aux mêmes valeurs propres $\lambda_{i}$. On en déduit que l'application $\widetilde{\Phi}$ n'est autre que celle étudiée en e) associée à la variété $(\widetilde{M}, \tilde{g})$ et aux fonctions propres $\tilde{f}_{i}$. $\widetilde{\Phi}$ doit donc être de degré \pm 1 , ce qui est contradictoire. 


\section{4. Équivariance de $\Phi$}

Nous allons exhiber, sous les hypothèses de la section 3, un morphisme du groupe Isom $M$ des isométries de $M$ dans le groupe $\mathrm{O}_{n+1}(\mathbb{R})$ des isométries de $\mathbb{S}^{n}$ pour lequel l'application $\Phi$ est équivariante.

Soient $f$ une fonction propre $(\triangle f=\lambda f)$ et $\sigma$ une isométrie de la variété $M$. Alors $\triangle(f \circ \sigma)=$ $\lambda(f \circ \sigma)$. En particulier, si on suppose que $\left(\sqrt{n+1} . f_{i}\right)_{1 \leqslant i \leqslant k}$ est une base de l'espace de toutes les fonctions propres associées aux valeurs propres de $M$ inférieures à un réel $\alpha$ donné, alors il existe une matrice $A^{\sigma}$ de $\mathcal{M}_{k}(\mathbb{R})$ telle que pour tout indice $i$, on ait $f_{i} \circ \sigma=\sum_{j} A_{i j}^{\sigma} f_{j}$. De plus, comme $\sigma$ préserve le volume riemannien, on a $\frac{\delta_{i j}}{n+1}=\frac{1}{\operatorname{Vol} M} \int_{M} f_{i} \circ \sigma \cdot f_{j} \circ \sigma d v_{g}=$ $\sum_{k l} A_{i k}^{\sigma} A_{j l}^{\sigma} \frac{\delta_{k l}}{n+1}$. On en déduit que $\sigma \mapsto A^{\sigma}$ est un morphisme de groupe de Isom $M$ à valeur dans $\mathrm{O}_{k}(\mathbb{R})$.

Dans le cas particulier où $M$ vérifie Ric $\geqslant(n-1)$ et $\lambda_{n+1} \leqslant n+\epsilon$, le choix d'une famille $\left(\sqrt{n+1} . f_{i}\right)_{1 \leqslant i \leqslant n+1} L^{2}$-orthonormée de fonctions propres associées aux $n+1$ premières valeurs propres non nulles de $M$ induit un morphisme de Isom $M$ à valeurs dans $\mathrm{O}(n+1)$ (d'après le lemme 10(i)) tel que $\Phi(\sigma(x))=A^{\sigma}(\Phi(x))$ pour toute isométrie $\sigma$. L'outil central de la démonstration des théorèmes 3 et 4 est le lemme suivant.

LEMME 16. - Soient $M$ une variété complète vérifiant $\mathrm{Ric} \geqslant n-1$ et $\lambda_{n+1} \leqslant n+\epsilon$ (pour $\epsilon \leqslant \alpha(n)$ ), et $\Phi: M \rightarrow \mathbb{S}^{n}$ l'application définie dans la Section 3. On se donne de plus $G$ un groupe fini d'isométries agissant librement sur M. Le morphisme $\sigma \mapsto A^{\sigma}$ est alors injectif sur $G$ et $A(G)$ est un sous-groupe de $\mathrm{O}_{n+1}(\mathbb{R})$ agissant librement sur $\mathbb{S}^{n}$. Enfin, $\Phi$ passe au quotient en une $C(n) \epsilon^{\frac{1}{384(n+1)^{3}}}$-approximation de Hausdorff de $M / G$ sur $\mathbb{S}^{n} / A(G)$.

Preuve. $-\operatorname{Si} \sigma \in \operatorname{Ker} A$, alors la variété quotient de $M$ par le groupe $\langle\sigma\rangle$ vérifie aussi Ric $\geqslant n-1$ et $\lambda_{n+1} \leqslant n+\epsilon$. Mais alors $M$ et $M /\langle\sigma\rangle$ ont des volumes presque égaux à celui de $\left(\mathbb{S}^{n}\right.$, can) (d'après 12). Or le cardinal de $\langle\sigma\rangle$ est égal au rapport de ces volumes (car $\langle\sigma\rangle$ agit librement), et donc $\operatorname{Ker} A=\left\{\operatorname{id}_{\mathrm{G}}\right\}$.

$G$ est un groupe agissant par isométrie sur $M$ et $\mathbb{S}^{n}$, et $\Phi$ est une application équivariante pour ces deux actions. $\Phi$ passe donc au quotient en une application de la variété riemannienne $M / G$ sur (l'orbifold) $\mathbb{S}^{n} / G$. Il est facile de montrer que $\Phi$ étant une $C(n) \epsilon^{\frac{1}{384(n+1)^{3}}}$-approximation de Hausdorff de $M$ sur $\mathbb{S}^{n}$ (proposition 12), son quotient est aussi une $C(n) \epsilon^{\frac{1}{384(n+1)^{3}}}$ approximation.

Pour montrer que $G$ agit librement sur $\mathbb{S}^{n}$, commençons par remarquer que, d'après le théorème de $\mathrm{J}$. Cheeger et $\mathrm{T}$. Colding cité en introduction, on peut supposer que sous les hypothèses du lemme, $M^{n}=\mathbb{S}^{n}$ (remarquez que dans la suite on a juste besoin que $M$ soit homéomorphe à $\mathbb{S}^{n}$, et donc [18] suffit). On utilise alors le résultat suivant.

LEMME 17. - Soit $G$ un groupe fini, soient $\alpha_{i}: G \times \mathbb{S}^{n} \rightarrow \mathbb{S}^{n}, i=1,2$, deux actions sur la sphère $\mathbb{S}^{n}$ et $\Phi:\left(\mathbb{S}^{n}, \alpha_{1}\right) \rightarrow\left(\mathbb{S}^{n}, \alpha_{2}\right)$ une application équivariante qui est de degré \pm 1 . Si $\alpha_{1}$ est libre, alors $\alpha_{2}$ est aussi libre.

Si $\alpha_{2}$ n'est pas libre, alors $G$ admet un élément non trivial $\sigma$ tel que $\alpha_{2}(\sigma)$ fixe un point de $\mathbb{S}^{n}$. Quitte à remplacer $G$ par $\left\langle\sigma^{l}\right\rangle$ (pour $l$ bien choisi), on peut se ramener au cas où $G$ est un groupe de cardinal $p$ premier fixant un point de $\mathbb{S}^{n}$ par l'action $\alpha_{2}$. Notons $M_{\Phi}$ le cylindre de l'application $\Phi$. Au couple $\left(M_{\Phi}, \mathbb{S}^{n}\right)$ est alors associée la suite longue exacte en cohomologie relative (à coefficients dans $\mathbb{Z} / p \mathbb{Z}$ ) suivante :

$$
\begin{aligned}
0 & \rightarrow H^{0}\left(M_{\Phi}, \mathbb{Z} / p \mathbb{Z}\right) \stackrel{\Phi^{*}}{\longrightarrow} H^{0}\left(\mathbb{S}^{n}, \mathbb{Z} / p \mathbb{Z}\right) \rightarrow H^{1}\left(M_{\Phi}, \mathbb{S}^{n} ; \mathbb{Z} / p \mathbb{Z}\right) \rightarrow \cdots \\
& \rightarrow H^{n}\left(M_{\Phi}, \mathbb{S}^{n} ; \mathbb{Z} / p \mathbb{Z}\right) \rightarrow H^{n}\left(M_{\Phi}, \mathbb{Z} / p \mathbb{Z}\right) \stackrel{\Phi^{*}}{\longrightarrow} H^{n}\left(\mathbb{S}^{n}, \mathbb{Z} / p \mathbb{Z}\right) \rightarrow 0
\end{aligned}
$$


Or, pour tout $1 \leqslant i<n$, on a $H^{i}\left(M_{\Phi}, \mathbb{Z} / p \mathbb{Z}\right)=H^{i}\left(\mathbb{S}^{n}, \mathbb{Z} / p \mathbb{Z}\right)=\{0\}$ et donc $H^{i}\left(M_{\Phi}, \mathbb{S}^{n}\right.$

$\mathbb{Z} / p \mathbb{Z})=\{0\}$. Enfin, puisque $\Phi$ est de degré \pm 1 , on en déduit que $\Phi^{*}$ est un isomorphisme entre $H^{n}\left(M_{\Phi}, \mathbb{Z} / p \mathbb{Z}\right)$ et $H^{n}\left(\mathbb{S}^{n}, \mathbb{Z} / p \mathbb{Z}\right)$ et donc :

$$
H^{i}\left(M_{\Phi}, \mathbb{S}^{n} ; \mathbb{Z} / p \mathbb{Z}\right)=\{0\}
$$

pour tout entier $i \geqslant 0$.

Comme $\Phi$ est équivariante, les actions $\alpha_{1}$ et $\alpha_{2}$ induisent une action $\alpha_{\Phi}$ sur $M_{\Phi}$ dont les points fixes $\operatorname{Fix}\left(\alpha_{\Phi}\right)$ s'identifient aux points fixes de $\alpha_{2}$ (car $\alpha_{1}$ est sans point fixe). D'après un théorème dû à E. Floyd (théorème 7.9, Chap. III, p. 144 de [5]), on a :

$$
\sum_{k \geqslant 0} \operatorname{dim} H^{k}(\underbrace{\operatorname{Fix}\left(\alpha_{\Phi}\right)}_{\operatorname{Fix}\left(\alpha_{2}\right)}, \underbrace{\operatorname{Fix}\left(\alpha_{1}\right)}_{\emptyset} ; \mathbb{Z} / p \mathbb{Z}) \leqslant \sum_{k \geqslant 0} \operatorname{dim} H^{k}\left(M_{\Phi}, \mathbb{S}^{n} ; \mathbb{Z} / p \mathbb{Z}\right)=0
$$

et donc $H^{k}\left(\operatorname{Fix}\left(\alpha_{2}\right), \emptyset ; \mathbb{Z} / p \mathbb{Z}\right)=H^{k}\left(\operatorname{Fix}\left(\alpha_{2}\right) ; \mathbb{Z} / p \mathbb{Z}\right)=\{0\}$ pour tout entier $k$, ce qui implique $\operatorname{Fix}\left(\alpha_{2}\right)=\emptyset$. D'où une contradiction (remarquez que dans le cas qui nous intéresse dans la suite, $\alpha_{2}$ agit par isométrie, et donc $\operatorname{Fix}\left(\alpha_{2}\right)$ ne pouvait être a priori que vide ou une sous-sphère).

\section{Démonstration des théorèmes 3 et 4}

Sous les hypothèses des théorèmes 3 et 4 , le revêtement riemannien universel (respectivement orientable) $(\widetilde{M}, \tilde{g})$ de la variété $M$ vérifie Ric $\geqslant n-1$ et est de volume presque égal à Vol $\mathbb{S}^{n}$. On en déduit que $(\widetilde{M}, \tilde{g})$ vérifie $\lambda_{n+1}(\widetilde{M}, \tilde{g}) \leqslant n+C(n)\left(\frac{\mathrm{Vol}^{n}}{2}-\mathrm{Vol} M\right)^{\beta(n)}$ (ce résultat découle qualitativement de P. Petersen [16] ; voir [3] pour une autre preuve et le calcul de la forme du majorant). Le lemme 16 implique alors que $\pi_{1}(M)$ (respectivement le groupe du revêtement orientable) agit librement sur $\mathbb{S}^{n}$ par isométrie et que le quotient de $\widetilde{\Phi}$ réalise une approximation de Hausdorff de $M$ (car le revêtement est normal) sur $\mathbb{S}^{n} / \pi_{1}(M)$ (respectivement $\mathbb{S}^{n} /(\mathbb{Z} / 2 \mathbb{Z})$ ). Pour la démonstration du théorème 3 , remarquons que le seul groupe d'isométries de cardinal 2 agissant librement sur $\mathbb{S}^{n}$ est $\{ \pm \mathrm{Id}\}$, et donc nos variétés sont proches de $\mathbb{P}^{n} \mathbb{R}$ en distance de Gromov-Hausdorff. Pour le théorème 4 , remarquons que le cardinal de $\pi_{1}(M)$ est exactement $k$ et donc que les variétés-quotient isométriques $\left(\mathbb{S}^{n}, c a n\right) / \pi_{1}(M)$ possibles sont en nombre fini (le nombre de classes d'équivalence des représentations dans $\mathrm{O}(n+1)$ des groupes finis de cardinal $k$ est fini ; voir [21, Chap. III] pour une classification des variétés complètes de courbure constante égale à 1 ). On en déduit que sous les hypothèses du théorème 4 , les variétés sont proches d'un nombre fini de variétés riemanniennes compactes possibles de même dimension. D'après le théorème de $\mathrm{J}$. Cheeger et $\mathrm{T}$. Colding cité en introduction, il existe une constante $\epsilon(n, k)>0$ telle que les variétés vérifiant les hypothèses du théorème 4 soient difféomorphes aux espaces quotients annoncés.

Nous finissons cette section par la preuve du corollaire 7. D'après le théorème de Lichnerowicz, on est ramené à considérer le cas où $\operatorname{Vol}(M) \# \pi_{1}(M) \geqslant(1-\delta(n)) \operatorname{Vol} \mathbb{S}^{n}$. Le revêtement riemannien de $(M, g)$ est alors encore une fois de volume presque égal à $\mathrm{Vol} \mathbb{S}^{n}$, et admet donc $n+1$ valeurs propres proches de $n$. $\mathrm{Si}\left(M^{n}, g\right)$ admettait au moins une valeur propre inférieure à $n+\delta(n)$, alors une fonction propre associée se releverait à $\widetilde{M}$ en une fonction propre qui pourrait être utilisée dans la construction de l'application $\widetilde{\Phi}$ du lemme 16. Mais, cette fonction propre de $\widetilde{M}$ étant invariante par l'action de $\pi_{1}(M)$, l'action de $\pi_{1}(M)$ sur $\mathbb{S}^{n}$ induite par $\widetilde{\Phi}$ admettrait un point fixe, ce qui impliquerait, d'après le lemme 16, que $\pi_{1}(M)$ est trivial. Ceci

$4^{\mathrm{e}}$ SÉRIE - TOME $38-2005-\mathrm{N}^{\circ} 3$ 
est contraire aux hypothèses du corollaire 7. L'énoncé sur le diamètre découle de celui sur le $\lambda_{1}$ et d'un résultat classique de $\mathrm{C}$. Croke [10] d'après lequel le $\lambda_{1}$ d'une variété de diamètre proche de $\pi$ et de courbure de Ricci minorée par $n-1$ est proche de $n$ (voir par exemple $[3,4]$ pour une redémonstration simple de ce fait).

\section{Démonstration du théorème 1}

Nous allons en fait montrer que, sur une variété vérifiant Ric $\geqslant n-1$, l'existence de $n$ valeurs propres proches de $n$ implique l'existence d'une $(n+1)$-ième valeur propre proche de $n$.

Nous commençons par supposer que $M$ est orientable. On munit $E$ (comme dans la section 3) de l'orientation compatible avec celle de $T M$. Si $\left(S_{1}, \ldots, S_{n}\right)$ est une famille de sections de $E$, on note $S=S_{1} \wedge \cdots \wedge S_{n}$ la section duale de la 1 -forme $S \mapsto \operatorname{det}\left(S_{1}, \ldots, S_{n}, S\right)$. $S_{1} \wedge \cdots \wedge S_{n}$ est bien évidemment $L^{2}$-orthogonale aux sections $S_{i}$ pour tout $i$. On a de plus les relations $D_{X}^{E}\left(S_{1} \wedge \cdots \wedge S_{n}\right)=\sum_{i=1}^{n} S_{1} \wedge \cdots \wedge D_{X}^{E} S_{i} \wedge \cdots \wedge S_{n}$ pour tout $X \in T M$ et $\left|S_{1} \wedge \cdots \wedge S_{n}\right|_{E} \leqslant\left|S_{1}\right|_{E} \cdots\left|S_{n}\right|_{E}$. On peut alors démontrer un premier résultat.

LEMME 18. - Il existe des fonctions $\alpha(n)>0$ et $C(n)>0$ (explicitement calculables) telles que si $M$ est orientable et vérifie $\lambda_{n}\left(\bar{\triangle}^{E}\right) \leqslant \epsilon$ (avec $\epsilon \leqslant \alpha(n)$ ), alors $\lambda_{n+1}\left(\bar{\triangle}^{E}\right) \leqslant C(n) \epsilon$.

Preuve.-Soient $\left(S_{1}, \ldots, S_{n}\right)$ une famille $L^{2}$-orthonormée de sections propres de $\triangle^{E}$ associées aux $n$ premières valeurs propres et $S_{n+1}=S_{1} \wedge \cdots \wedge S_{n}$. Le lemme 11(iii) nous donne :

$$
\left\|D^{E} S_{n+1}\right\|_{2}^{2} \leqslant n^{2}\left(\max _{i \leqslant n}\left\|S_{i}\right\|_{\infty}\right)^{2 n-2} \max _{i \leqslant n}\left\|D^{E} S_{i}\right\|_{2}^{2} \leqslant C(n) \epsilon .
$$

Montrons que la norme $L^{2}$ de $S_{n+1}$ est proche de $1:$ d'après la remarque qui suit le lemme 11, on a $\left\|S_{n+1}\right\|_{\infty} \leqslant \prod_{i \leqslant n}\left\|S_{i}\right\|_{\infty} \leqslant 1+C(n) \sqrt{\epsilon}$, et il existe un sous-ensemble $M_{\epsilon}$ de $M$ tel que :

$$
\left|\left\langle S_{i}, S_{j}\right\rangle_{E}-\delta_{i j}\right| \leqslant C(n) \epsilon^{1 / 4}, \quad \operatorname{Vol} M_{\epsilon} \geqslant\left(1-C(n) \epsilon^{1 / 4}\right) \operatorname{Vol} M .
$$

Fixons un point $x$ de $M$ et $\left(X_{1}, \ldots, X_{n+1}\right)$ un repère orthonormé direct de $E_{x}$ tel que $\operatorname{Vect}\left(X_{1}, \ldots, X_{n}\right)$ et $\operatorname{Vect}\left(S_{1}(x), \ldots, S_{n}(x)\right)$ coüncident; on a alors $S_{1} \wedge \cdots \wedge S_{n}=c X_{n+1}$, où $c^{2}$ vaut :

$$
\operatorname{det}\left(\begin{array}{ccc}
\left\langle S_{1}, S_{1}\right\rangle_{E} & \ldots & \left\langle S_{n}, S_{1}\right\rangle_{E} \\
\left.\right|_{\left\langle S_{1}, S_{n}\right\rangle_{E}} & \ldots & \left\langle S_{n}, S_{n}\right\rangle_{E}
\end{array}\right)
$$

On en déduit que :

$$
\left.|| S_{n+1}(x)\right|^{2}-1|=| c^{2}-1|=| \operatorname{det}\left(\left\langle S_{i}, S_{j}\right\rangle_{E}\right)_{i j}-\operatorname{det} I_{n} \mid \leqslant C(n) \epsilon^{1 / 4},
$$

en tout point $x$ de $M_{\epsilon}$. On obtient donc :

$$
\begin{aligned}
\left|\left\|S_{n+1}\right\|_{2}^{2}-1\right| & \leqslant\left|\frac{1}{\operatorname{Vol} M} \int_{M_{\epsilon}}\left(\left|S_{n+1}\right|^{2}-1\right)\right|+\left|\frac{1}{\operatorname{Vol} M} \int_{M \backslash M_{\epsilon}}\left(\left|S_{n+1}\right|^{2}-1\right)\right| \\
& \leqslant C(n) \epsilon^{1 / 4}+\left(1-\frac{\operatorname{Vol} M_{\epsilon}}{\operatorname{Vol} M}\right) 2 C(n) \leqslant C(n) \epsilon^{1 / 4} .
\end{aligned}
$$


$\left(S_{i}\right)_{1 \leqslant i \leqslant n+1}$ est donc $L^{2}$ presque othonormée et $\left\|D^{E} S_{i}\right\|_{2}^{2} \leqslant C(n) \epsilon$ pour tout $1 \leqslant i \leqslant n+1$. Par l'inégalité de Cauchy-Schwarz, on obtient que pour toute combinaison linéaire $S$ des sections $S_{1}, \ldots, S_{n}$ et $S_{n+1}$ on a $\left\|D^{E} S\right\|_{2}^{2} \leqslant C(n) \epsilon\|S\|_{2}^{2}$. Le principe du min-max conclut.

On peut alors en déduire la proposition suivante (remarquer qu'on ne suppose plus que $M$ est orientable) qui combinée à la proposition 12 et au theorème de J. Cheeger et T. Colding (cité en introduction) démontre le théorème 1 .

Proposition 19. - Il existe des constantes $\alpha(n)>0$ et $C(n)>0$ (universellement calculables) telles que si $M$ vérifie $\lambda_{n}(M) \leqslant n+\epsilon$ (avec $\epsilon \leqslant \alpha(n)$ ), alors $\lambda_{n+1}(M) \leqslant$ $n+C(n) \epsilon^{1 / 2}$.

Remarque. - Bien évidemment, on peut décliner cette proposition en remplaçant la conclusion par l'une des inégalités :

$$
\begin{aligned}
& \operatorname{Rad} M \geqslant\left(1-C(n) \epsilon^{\beta(n)}\right) \pi, \quad \operatorname{Vol} M \geqslant\left(1-C(n) \epsilon^{\beta(n)}\right) \operatorname{Vol} \mathbb{S}^{n} \\
& \quad \text { ou } d_{G H}\left(M, \mathbb{S}^{n}\right) \leqslant C(n) \epsilon^{\beta(n)}
\end{aligned}
$$

où $C(n)$ et $\beta(n)$ sont des constantes explicitement calculables.

Preuve. - Si $M$ est orientable, la proposition découle directement des propositions 10(i) et (ii) et du lemme 18. Supposons donc que $M$ n'est pas orientable. Soit $\left(\sqrt{n+1} \cdot f_{i}\right)_{1 \leqslant i \leqslant n}$ une famille $L^{2}$-orthonormée de fonctions propres associées aux $n$ premières valeurs propres non nulles de $M$ et soit $\pi:\left(\widetilde{M}^{n}, \tilde{g}\right) \rightarrow M$ le revêtement riemannien orientable de $M$; notons $\tilde{f}_{i}=f_{i} \circ \pi$. Sur $\left(\widetilde{M}^{n}, \tilde{g}\right)$ on obtient une fonction $\tilde{f}_{n+1}$ telle que $\left(\sqrt{n+1} \cdot \tilde{f}_{i}\right)_{i \leqslant n+1}$ soit une famille de fonctions propres de $(\widetilde{M}, \tilde{g})$ associées à des valeurs propres proches de $n$. Le morphisme de groupe $A$ de $\operatorname{Gr}(\widetilde{M}, M)$ dans $\mathrm{O}_{n+1}(\mathbb{R})$ construit à partir de la famille $\left(\tilde{f}_{i}\right)_{i \leqslant n+1}$ (voir le lemme 16) est injectif et $A(\operatorname{Gr}(\widetilde{M}, M))$ agit librement sur $\mathbb{R}^{n+1}$. Or, l'hyperplan engendré par $\left(\tilde{f}_{i}\right)_{1 \leqslant i \leqslant n}$ est évidemment fixé par $\operatorname{Gr}(\widetilde{M}, M)$, ce qui est contradictoire.

Remarque. - La preuve de la proposition 19 peut se faire sans théorie de Flyod : on sait que l'élément non trivial de $\operatorname{Gr}(\widetilde{M}, M)$ est d'ordre 2, fixe l'hyperplan engendré par les fonctions $\left(f_{i} \circ \pi\right)$ et donc agit par symétrie orthogonale hyperplane sur $\mathbb{S}^{n}$. On en déduit que l'application $\widetilde{\Phi}$ de degré \pm 1 construite à partir des fonctions propres $\left(\tilde{f}_{i}\right)_{i \leqslant n+1}$ passe au quotient en une application $\Phi$ de $M$ sur la demi-sphère $\frac{1}{2} \mathbb{S}^{n}$ dont le degré modulo 2 est égal à 1 . Or $M$ est une variété sans bord et $\frac{1}{2} \mathbb{S}^{n}$ est une variété à bord (et même contractile), le degré modulo 2 de $\Phi$ ne peut donc être que nul, d'où une contradiction.

\section{Optimalité du théorème 1}

Pour clore cet article, nous allons montrer que pour tout entier $p \in\{1, \ldots, n-1\}$, il existe une suite $\left(g_{k}\right)_{k \in \mathbb{N}}$ de métriques sur $\mathbb{S}^{n}$ qui vérifie Ric $\geqslant(n-1), \lambda_{p}\left(g_{k}\right) \rightarrow n, \lambda_{p+1}\left(g_{k}\right) \rightarrow \beta(n)>n$, $\operatorname{Vol}\left(g_{k}\right) \rightarrow 0,\left(\mathbb{S}^{n}, g_{k}\right)$ tend en distance de Gromov-Hausdorff vers la demi-sphère canonique de dimension $p$ et par conséquent, la suite des rayons couvrants tend vers $\frac{\pi}{2}$ (ce qui montre qu'aucun des résultats de stabilité obtenus dans la proposition 19 et dans la remarque qui la suit ne se généralise sous l'hypothèse $\lambda_{n-1} \leqslant n+\epsilon$ ). En revanche, nous n'avons pas encore réussi à construire une suite de variétés non difféomorphes à $\mathbb{S}^{n}$ de courbure de Ricci supérieure à $(n-1)$ et telle que la suite des $\lambda_{n-1}$ tende vers $n$ (voir [1,15] pour des variétés n'ayant pas le même type d'homotopie que $\mathbb{S}^{n}$, qui supportent des métriques dont la courbure de Ricci est supérieure à $(n-1)$ et dont une seule valeur propre est arbitrairement proche de $n$ ).

$4^{\text {e }}$ SÉRIE - TOME $38-2005-\mathrm{N}^{\circ} 3$ 
Exemple. - La métrique $g_{k}$ est une généralisation des fuseaux en codimension quelconque. En fait, on construit $g_{k}$ en écrasant la métrique canonique de $\mathbb{S}^{n}$ dans le fibré normal à une soussphère $\mathbb{S}^{p-1}$. La difficulté étant de régulariser la métrique sur $\mathbb{S}^{p-1}$ et sur son cut-locus $\mathbb{S}^{n-p}$ tout en préservant le minorant sur la courbure de Ricci. Nous décrivons la suite $g_{k}$ dans le cas particulier $p=n-1$; la généralisation aux autres valeurs de $p$ ne pose aucun problème (notons que dans le cas $p=n$, il n'est pas possible d'écraser la métrique dans le facteur normal). Soit $k$ un entier non nul. Nous commençons par fixer les notations suivantes :

$$
\begin{gathered}
\eta_{k}=\sqrt{\sin ^{2}\left(\frac{1}{\sqrt{k}}\right)+\frac{1}{k^{2}} \cos ^{2}\left(\frac{1}{\sqrt{k}}\right)}, \quad \epsilon_{k}=\frac{\frac{\pi}{2}-\frac{1}{\sqrt{k}}}{k}, \\
\theta_{k}=\arctan \left(\frac{1}{k \tan \frac{1}{\sqrt{k}}}\right)-\frac{\pi}{2 k}+\frac{1}{k \sqrt{k}} .
\end{gathered}
$$

On note $I_{k}$ l'intervalle $] 0, \frac{\pi}{2}-\theta_{k}\left[\right.$. Sur la variété $M=I_{k} \times \mathbb{S}^{1} \times \mathbb{S}^{n-2}$, on considère la métrique $g_{k}=d r^{2}+a_{k}(r)^{2} g_{\mathbb{S}^{1}}+b_{k}(r)^{2} g_{\mathbb{S}^{n-2}}$, où $g_{\mathbb{S}^{1}}$ et $g_{\mathbb{S}^{n-2}}$ sont les métriques canoniques des sphères $\mathbb{S}^{1}$ et $\mathbb{S}^{n-2}$, et où $a_{k}$ et $b_{k}$ sont des fonctions définies sur $I_{k}$ par les formules :

$$
a_{k}(r)= \begin{cases}\frac{1}{k} \sin (k r) & \left.\operatorname{sur}] 0, \epsilon_{k}\right], \\ \eta_{k} \sin \left(r+\theta_{k}\right) & \operatorname{sur}\left[\epsilon_{k}, \frac{\pi}{2}-\theta_{k}[\right.\end{cases}
$$

et

$$
b_{k}(r)= \begin{cases}\frac{\epsilon_{k}}{\epsilon_{k}+\theta_{k}} \cos \left(\left(\frac{\epsilon_{k}+\theta_{k}}{\epsilon_{k}}\right) r\right)+\frac{\theta_{k}}{\epsilon_{k}+\theta_{k}} \cos \left(\theta_{k}+\epsilon_{k}\right) & \text { sur } \left.] 0, \epsilon_{k}\right], \\ \cos \left(r+\theta_{k}\right) & \text { sur }\left[\epsilon_{k}, \frac{\pi}{2}-\theta_{k}[.\right.\end{cases}
$$

Remarquons que la fonction $a_{k}$ (respectivement $b_{k}$ ) est $C^{1}$ sur $I_{k}, C^{2}$ en dehors de $\epsilon_{k}$ et tend vers 0 en 0 (respectivement tend vers 0 en $\frac{\pi}{2}-\theta_{k}$ ). La métrique $g_{k}$ se prolonge en une métrique $C^{1}$ sur $\mathbb{S}^{n}$ pour laquelle le cercle $\mathbb{S}^{1}$ est le cut-locus de la sous-sphère $\mathbb{S}^{n-2}$.

Pour minorer la courbure de Ricci, on applique les formules de calcul de la courbure de Ricci des doubles-produits tordus (voir par exemple [15]). En un point $x$ fixé de $M$ on note $\frac{\partial}{\partial r}$ un vecteur tangent au facteur $I_{k}, u$ un élément de $T_{x} \mathbb{S}^{1}, v$ un élément de $T_{x} \mathbb{S}^{n-2}$. Alors, la courbure de $\operatorname{Ricci~} \operatorname{Ric}_{k}$ de $\left(\mathbb{S}^{n}, g_{k}\right)$ vérifie les propriétés suivantes :

$$
\begin{aligned}
& \operatorname{Ric}_{k}\left(\frac{\partial}{\partial r}, u\right)=\operatorname{Ric}_{k}\left(\frac{\partial}{\partial r}, v\right)=\operatorname{Ric}_{k}(u, v)=0, \\
& \operatorname{Ric}_{k}\left(\frac{\partial}{\partial r}, \frac{\partial}{\partial r}\right)=-\frac{a^{\prime \prime}}{a}-(n-2) \frac{b^{\prime \prime}}{b} \\
& =\left\{\begin{array}{l}
\left.\left.k^{2}+(n-2)\left(\frac{\epsilon_{k}+\theta_{k}}{\epsilon_{k}}\right)^{2} \frac{\cos \left(\left(\frac{\epsilon_{k}+\theta_{k}}{\epsilon_{k}}\right) r\right)}{\cos \left(\left(\frac{\epsilon_{k}+\theta_{k}}{\epsilon_{k}}\right) r\right)+\frac{\theta_{k}}{\epsilon_{k}} \cos \left(\theta_{k}+\epsilon_{k}\right)} \geqslant k^{2} \quad \text { sur }\right] 0, \epsilon_{k}\right], \\
n-1 \quad \operatorname{sur}\left[\epsilon_{k}, \frac{\pi}{2}-\theta_{k}[,\right.
\end{array}\right. \\
& \frac{\operatorname{Ric}_{k}(u, u)}{g_{k}(u, u)}=-\frac{a^{\prime \prime}}{a}-(n-2) \frac{a^{\prime} b^{\prime}}{a b} \\
& =\left\{\begin{array}{l}
\left.\left.k^{2}+(n-2) \frac{k \cos (k r)}{\sin (k r)}\left(\frac{\epsilon_{k}+\theta_{k}}{\epsilon_{k}}\right) \frac{\sin \left(\left(\frac{\epsilon_{k}+\theta_{k}}{\epsilon_{k}}\right) r\right)}{\cos \left(\left(\frac{\epsilon_{k}+\theta_{k}}{\epsilon_{k}}\right) r\right)+\frac{\theta_{k}}{\epsilon_{k}} \cos \left(\theta_{k}+\epsilon_{k}\right)} \geqslant k^{2} \quad \text { sur }\right] 0, \epsilon_{k}\right], \\
n-1 \quad \operatorname{sur}\left[\epsilon_{k}, \frac{\pi}{2}-\theta_{k}[\right.
\end{array}\right.
\end{aligned}
$$


et

$$
\frac{\operatorname{Ric}_{k}(v, v)}{g_{k}(v, v)}=-\frac{b^{\prime \prime}}{b}-\frac{a^{\prime} b^{\prime}}{a b}+(n-3)\left(\frac{1-b^{\prime 2}}{b^{2}}\right)
$$

Donc

$$
\begin{aligned}
\frac{\operatorname{Ric}_{k}(v, v)}{g_{k}(v, v)} & \geqslant\left(\frac{\epsilon_{k}+\theta_{k}}{\epsilon_{k}}\right)^{2} \frac{\cos \left(\left(\frac{\epsilon_{k}+\theta_{k}}{\epsilon_{k}}\right) r\right)}{\cos \left(\left(\frac{\epsilon_{k}+\theta_{k}}{\epsilon_{k}}\right) r\right)+\frac{\theta_{k}}{\epsilon_{k}} \cos \left(\theta_{k}+\epsilon_{k}\right)} \\
& \left.\left.\geqslant \frac{\epsilon_{k}+\theta_{k}}{\epsilon_{k}} \sim \frac{2}{\pi} \sqrt{k} \quad \text { sur }\right] 0, \epsilon_{k}\right]
\end{aligned}
$$

$\left(\operatorname{car} \theta_{k}+\epsilon_{k} \geqslant\left(\frac{\epsilon_{k}+\theta_{k}}{\epsilon_{k}}\right) r\right)$ et

$$
\frac{\operatorname{Ric}_{k}(v, v)}{g_{k}(v, v)}=2+(n-3) \frac{1-\sin ^{2}\left(r+\theta_{k}\right)}{\cos ^{2}\left(r+\theta_{k}\right)}=n-1 \quad \operatorname{sur}\left[\epsilon_{k}, \frac{\pi}{2}-\theta_{k}[.\right.
$$

On déduit des calculs précédents que $\left(\mathbb{S}^{n}, g_{k}\right)$ est de courbure de Ricci supérieure à $(n-1)$ pour $k$ assez grand.

Il est évident que le volume des métriques $g_{k}$ tend vers 0 . De plus, la suite de variétés ainsi obtenue tend (en distance de Gromov-Hausdorff) vers une des hémisphères de dimension $n-1$ que borde la sphère $\mathbb{S}^{n-2}$ : notons $\left(N_{k}, h_{k}\right)$ la variété $] \epsilon_{k}, \frac{\pi}{2}-\theta_{k}\left[\times \mathbb{S}^{n-2}\right.$, munie de la métrique $h_{k}=(d r)^{2}+\cos ^{2}\left(r+\theta_{k}\right) g_{\mathbb{S}^{n-2}} ;$ elle est isométrique à une boule géodésique de $\mathbb{S}^{n-1}$ de rayon $\frac{\pi}{2}-\left(\epsilon_{k}+\theta_{k}\right)$ (privée de son centre), donc elle converge vers la demi-sphère $\frac{1}{2} \mathbb{S}^{n-1}$, munie de sa métrique canonique. Par ailleurs, $\left(N_{k}, h_{k}\right)$ se plonge dans $\left(M, g_{k}\right)$ de manière isométrique, via l'application $(r, v) \mapsto\left(r, u_{0}, v\right)$, où $u_{0}$ est un point fixé de $\mathbb{S}^{1}$. De plus, la distance dans $M$ entre deux points $p=\left(r, u_{0}, v\right)$ et $q=\left(r^{\prime}, u_{0}, v^{\prime}\right)$ coïncide avec la distance dans $N_{k}$ entre $(r, v)$ et $\left(r^{\prime}, v^{\prime}\right)$. Comme $d_{g_{k}}\left[(r, u, v) ;\left(r, u_{0}, v\right)\right] \leqslant \pi \eta_{k}$, on obtient que $\left(M, g_{k}\right)$ converge vers l'hémisphère de $\mathbb{S}^{n-1}$ au sens de Gromov-Hausdorff. Il nous reste à montrer que le laplacien de ces variétés admet au moins $n-1$ valeurs propres proches de $n$. Pour cela, nous pouvons soit appliquer un résultat de J. Bertrand [4], soit choisir des points $x_{0}, \ldots, x_{n-2}$ sur $\mathbb{S}^{n-2}$ de sorte que $d_{\mathbb{S}^{n-2}}\left(x_{i}, x_{j}\right)=\frac{\pi}{2}$ si $i \neq j$ et noter encore $x_{i}$ le point $\left(0, u_{0}, x_{i}\right)$ de $M$; en appliquant le principe du min-max aux $(n-1)$ fonctions $f_{i}(x)=\cos \left(d_{k}\left(x_{i}, x\right)\right)$ (la forme des métriques $g_{k}$ fait que les intégrales à calculer convergent vers les intégrales correspondantes calculées sur $\left(\frac{1}{2} \mathbb{S}^{n-1}\right.$, can $)$; voir [3] pour les détails). Enfin, les métriques $g_{k}$ n'ont pas plus de $n-1$ valeurs propres proches de $n$ car sinon, d'après [4], les variétés $\left(\mathbb{S}^{n}, g_{k}\right)$ contiendraient une sous-partie Gromov-Hausdorff proche de $\left(\mathbb{S}^{n-1}\right.$, can $)$; or une demi-sphère de dimension $n-1$ ne peut contenir une partie Gromov-Hausdorff proche de la sphère $\left(\mathbb{S}^{n-1}, \mathrm{can}\right)$.

À propos de ces exemples, remarquer que la $l$-ième valeur propre $\lambda_{l}^{k}$ de la variété $\left(M^{n}, g_{k}\right)$ reste bornée par une fonction de $l, n$ et $p$ (pour $l$ quelconque) lorsque $k$ tend vers l'infini car les fonctions propres radiales pour le problème de Neuman de la demi-sphère de dimension $p-1$ permettent de construire, comme précédemment, une famille presque $L^{2}$-orthonormée de fonctions tests sur $M$ dont les quotients de Rayleigh pour la métrique $g_{k}$ tendent vers le spectre pour le problème de Neuman de la demi-sphère de dimension $p-1$. On conclut alors par le principe du min-max.

\section{Remerciements}

Je remercie S. Gallot pour ses nombreux encouragements et U. Suter pour les nombreuses conversations concernant le lemme 17.

$4^{\mathrm{e}}$ SÉRIE - TOME $38-2005-\mathrm{N}^{\circ} 3$ 


\section{RÉFÉRENCES}

[1] Anderson M., Metrics of positive Ricci curvature with large diameter, Manuscripta Math. 68 (1990) $405-415$.

[2] Aubry E., Théorème de la Sphère, Séminaire de Théorie Spectrale et Géométrie, Grenoble 18 (2000) 125-155.

[3] AubRY E., Variétés de courbure de Ricci presque minorée : inégalités géométriques optimales et stabilité des variétés extrémales, Thèse, Institut Fourier, Grenoble, 2003.

[4] Bertrand J., Pincement spectral en courbure de Ricci positive, Prépublications de l'École polytechnique, $\mathrm{n}^{\mathrm{O}} \mathbf{2 0 0 3 - 1 3}$.

[5] Bredon G., Introduction to Compact Transformation Groups, Pure Appl. Math., vol. 46, Academic Press, New York, 1972.

[6] Cheeger J., Colding T., On the structure of spaces with Ricci curvature bounded below. I, J. Differential Geom. 46 (1997) 406-480.

[7] Cheeger J., Colding T., On the structure of spaces with Ricci curvature bounded below. III, J. Differential Geom. 52 (1999) 37-74.

[8] Colding T., Shape of manifolds with positive Ricci curvature, Invent. Math. 124 (1996) 175-191.

[9] Colding T., Large manifolds with positive Ricci curvature, Invent. Math. 124 (1996) 193-214.

[10] Croke C., An eigenvalue pinching theorem, Invent. Math. 68 (1982) 253-256.

[11] Gallot S., Courbure de Ricci et convergence des variétés, in : Colding T.H., Cheeger-Colding (Eds.), Séminaire Bourbakih, Nov. 1997 (exposé no 835), in: Astérisque, vol. 252, 1998, pp. 7-32.

[12] Gromov M., Metric Structures for Riemannian and Non-Riemannian Spaces, Progr. Math., vol. 152, Birkhäuser, Boston, 1999.

[13] IKedA A., On the spectrum of a Riemannian manifold of positive constant curvature, Osaka J. Math. 17 (1980) 75-93.

[14] ILIAS S., Constantes explicites pour les inégalités de Sobolev sur les variétés riemanniennes compactes, Ann. Inst. Fourier 33 (1983) 151-165.

[15] ОтsU Y., On manifolds of positive Ricci curvature with large diameter, Math. Z. 206 (1991) 252-264.

[16] Petersen P., On eigenvalue pinching in positive Ricci curvature, Invent. Math. 138 (1999) 1-21.

[17] Petersen P., On eigenvalue pinching in positive Ricci curvature, Erratum, Invent. Math. 155 (2004) 223.

[18] Perelman G., Manifold of positive Ricci curvature with almost maximal volume, J. Amer. Math. Soc. 7 (1994) 299-305.

[19] Ruh E., Curvature and differentiable structure on spheres, Bull. Amer. Math. Soc. 77 (1971) 148-150.

[20] SAKAI T., Riemannian Geometry, American Mathematical Society, Providence, RI, 1996.

[21] Wolf J.A., Spaces of Constant Curvature, Publish or Perish, Wilmington, DE, 1984.

[22] Yamaguchi T., Lipschitz convergence of manifolds of positive Ricci curvature with large volume, Math. Ann. 284 (1989) 423-436. 